\title{
Evaluation of Radiochemical Data Usability
}

\author{
J. G. Paar \\ University of Tennessee, Knoxville/Oak Ridge National Laboratory \\ D. R. Porterfield \\ Chemical Science and Technology Division \\ Los Alamos National Laboratory \\ Environmental Restoration Division \\ P.O. Box 2003 \\ Oak Ridge, Tennessee 37831-7294
}

Date Issued-April 1997

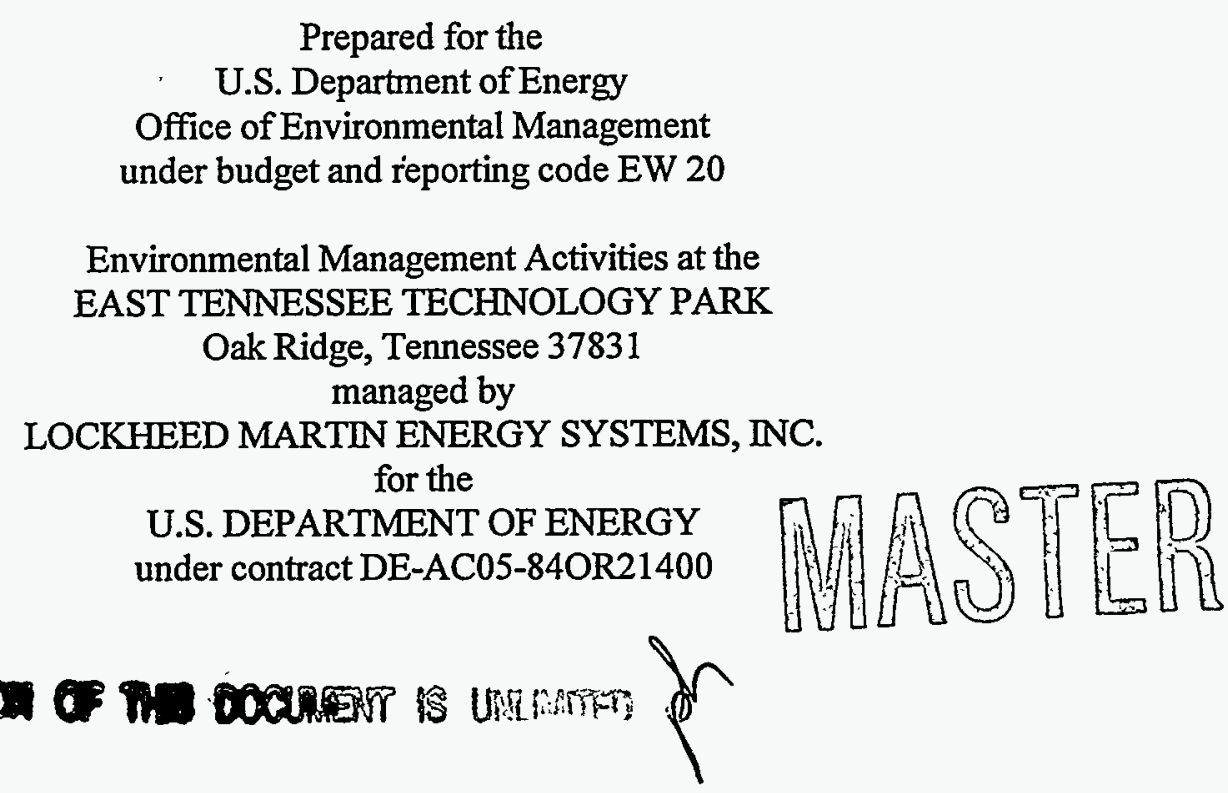

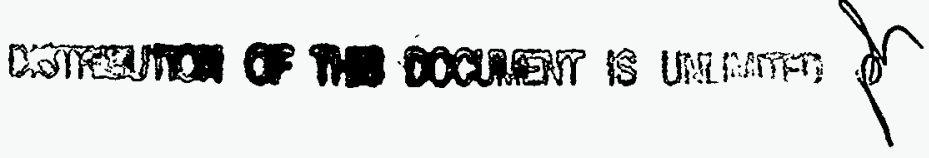




\section{DISCLAMERR}

Portions of this document may be illegible in electronic image products. Images are produced from the best available original document. 


\section{DISCLAIMER}

This report was prepared as an account of work sponsored by an agency of the United States Government. Neither the United States Government nor any agency thereof, nor any of their employees, make any warranty, express or implied, or assumes any legal liability or responsibility for the accuracy, completeness, or usefulness of any information, apparatus, product, or process disclosed, or represents that its use would not infringe privately owned rights. Reference herein to any specific commercial product, process, or service by trade name, trademark, manufacturer, or otherwise does not necessarily constitute or imply its endorsement, recommendation, or favoring by the United States Government or any agency thereof. The views and opinions of authors expressed herein do not necessarily state or reflect those of the United States Government or any agency thereof. 


\section{PREFACE}

Environmental remedial decisions require the acquisition of data of known and sufficient quality for intended use. Data verification and validation (V\&V) are two of the tools that ensure a level of quality assurance in data usability. When applied to radiochemistry, no national standard currently covers V\&V concepts adequately, and the need for a document of this type was recognized by most of the Department of Energy Complex. This document was developed through intersite cooperation and provides a reasonable approach for the evaluation of radioanalytical data for purposes of environmental remediation but can also be applied to data intended for non-remedial purposes.

This document is intended to provide a framework onto which implementing procedures can be written. It is a stand-alone document for the purposes of data evaluation; however, sufficient laboratory deliverables must exist to enable the V\&V tools to be used. It is recommended that if $\mathrm{V} \& \mathrm{~V}$ procedures are written based on this document, that corresponding specifications for analytical laboratory deliverables are also written. 



\section{ACKNOWLEDGMENTS}

Many persons were of great help in developing this document and are listed below. As this document is not a "consensus standard," consensus is not implied in listing these names. The intention here is to thank all those who helped in developing this document.

$\begin{array}{ll}\text { D. Bottrell } & \text { DOE-EM 263 } \\ \text { D. Carden } & \text { DOE-ORO } \\ \text { J. Chambers } & \text { FERMCO } \\ \text { B. Crandall } & \text { WSRC } \\ \text { A. Daniel } & \text { MMES } \\ \text { J. Fisk } & \text { LANL } \\ \text { J. Griggs } & \text { EPA-NAREL } \\ \text { L. Hook } & \text { MMES } \\ \text { J. Karnofsky } & \\ \text { T. Killeen } & \text { WSRC } \\ \text { C.K. Liu } & \text { DOE-RL } \\ \text { D. McCurdy } & \text { Yankee Atomic } \\ \text { M. Miller } & \text { EQM } \\ \text { D. Porterfield } & \text { LANL } \\ \text { M. Ridley } & \text { LLNL } \\ \text { M. Schwab } & \text { BHC } \\ \text { J. Warren } & \text { EPA/QAMS } \\ \text { R. Wells } & \text { INEL } \\ \text { L. Withrow } & \text { Quanterra } \\ \text { C. Wrotriiak } & \text { Dames \& Moore }\end{array}$





\section{CONTENTS}

PREFACE $\ldots \ldots \ldots \ldots \ldots \ldots \ldots \ldots \ldots \ldots \ldots \ldots \ldots \ldots \ldots \ldots$

ACKNOWLEDGMENTS $\ldots \ldots \ldots \ldots \ldots \ldots \ldots \ldots \ldots \ldots \ldots \ldots \ldots \ldots$

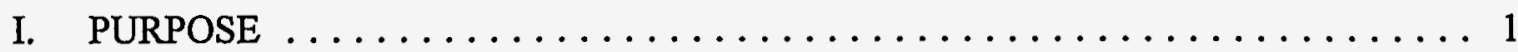

II. REFERENCES $\ldots \ldots \ldots \ldots \ldots \ldots \ldots \ldots \ldots \ldots \ldots \ldots \ldots \ldots \ldots \ldots$

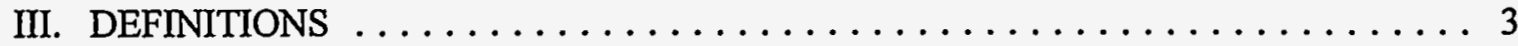

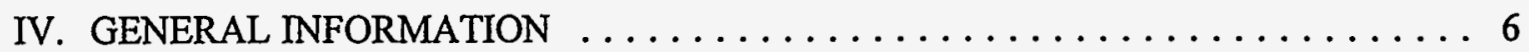

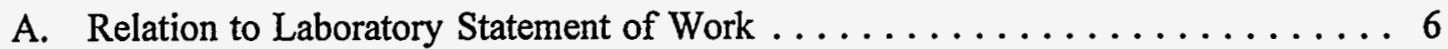

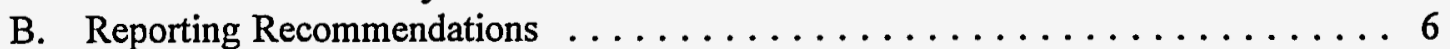

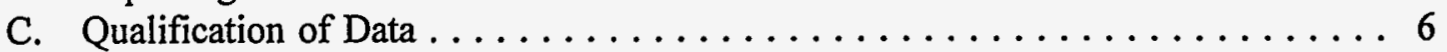

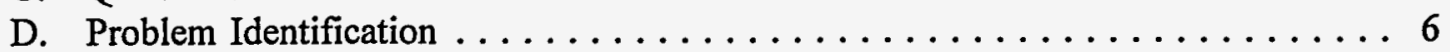

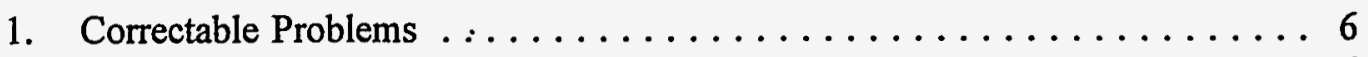

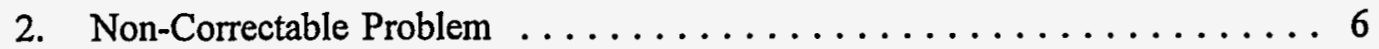

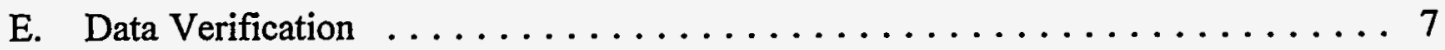

1. Verification Report ...................... 7

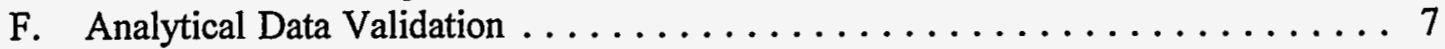

1. Validation Report ....................... 7

V. PROCEDURE ...........................

A. Custody of Samples and Sample Documentation $\ldots \ldots \ldots \ldots \ldots \ldots \ldots$

1. Verification .................... 8

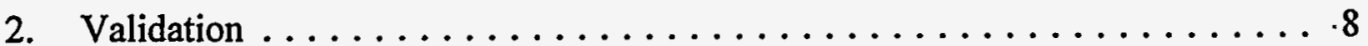

B. Holding Time and Turn-around Time $\ldots \ldots \ldots \ldots \ldots \ldots \ldots \ldots \ldots$

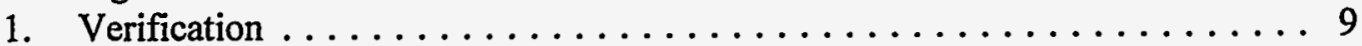

2. Validation ............................. 9

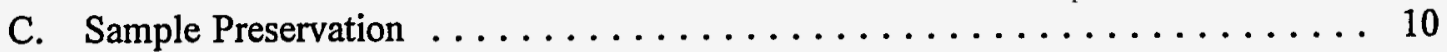

1. Verification .......................... 10

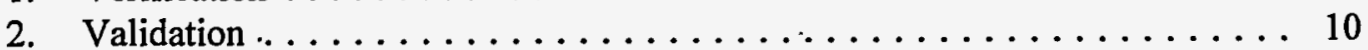

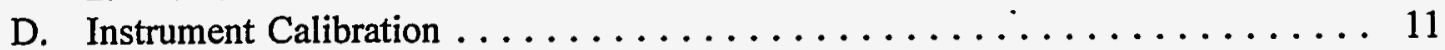

1. Scope . . . . . . . . . . . . . . . . . . . . 11

2. Mechanism for Initial Calibration Evaluation ............... 11

3. General Technical Specifications for Initial Calibration . . . . . . . . 11

4. Specific Technical Specifications for Calibration Verification $\ldots \ldots \ldots .13$

5. Initial Calibration for Gross Alpha and Gross Beta

by Gas Proportional Systems ................... 14

6. Calibration Verification for Gross Alpha and Gross Beta

by Gas Proportional Systems . . . . . . . . . . . . . 15

7. Initial Calibration for Liquid Scintillation $\ldots \ldots \ldots \ldots \ldots \ldots \ldots \ldots$

8. Calibration Verification for Liquid Scintillation . . . . . . . . . 18

9. Initial Calibration for Alpha Spectrometry . . . . . . . . . . . 20

10. Calibration Verification for Alpha Spectrometry $\ldots \ldots \ldots \ldots 21$

vii 
11. Initial Calibration for Gamma Spectrometry $\ldots \ldots \ldots \ldots \ldots . \ldots . \ldots . \ldots . . \ldots 23$

12. Calibration Verification for Gamma Spectrometry ............ 24

E. Quality-indicator samples . . . . . . . . . . . . . . . . . . 26

1. Total Propagated Uncertainty (TPU) . . . . . . . . . . . . . 27

2. Standard Traceability . . . . . . . . . . . . . . . . 28

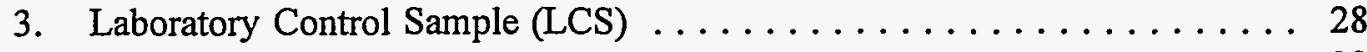

4. Laboratory Duplicate . . . . . . . . . . . . . . . . . 29

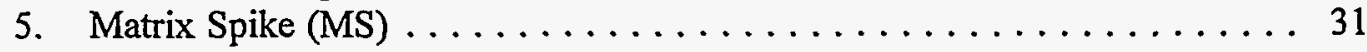

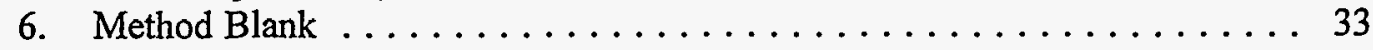

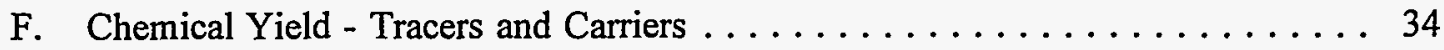

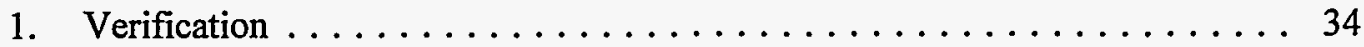

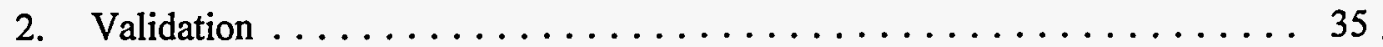

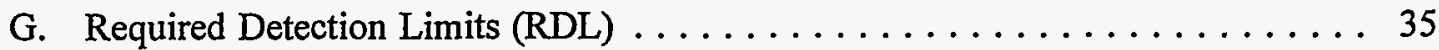

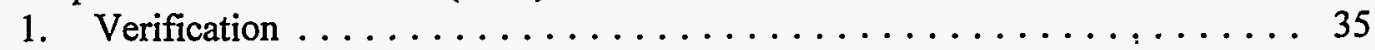

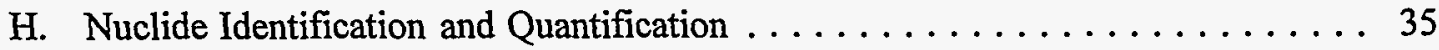

1. Test for Detection ....................... 35

2. Negative Results ...................... 36

3. Sample Result Recalculation . . . . . . . . . . . . . 36

I. Instrument-Specific Sample Considerations $\ldots \ldots \ldots \ldots \ldots \ldots \ldots \ldots \ldots \ldots$

1. Sample Analytical Parameters for Gas Proportional Counting . . . . . . . 37

2. Spectral Interpretation (alpha and gamma spectrometry) . . . . . . . 37

VI. APPENDIXES ........................... 39

APPENDIX A: DATA VALIDATION QUALIFIERS AND REASON CODES . . . . . A-1

APPENDIX B: MAPPING FOR MULTIPLE QUALITY DEFICIENCIES . . . . . . B-1

APPENIDX C: $\quad$ CALCULATIONS AND EQUATIONS $\ldots \ldots \ldots \ldots \ldots \ldots \ldots$ C-1

APPENDIX D: LISTING OF COMMON NUCLIDES/METHODOLOGIES . . . . . . D-1

APPENDIX E: RECOMMENDED SURVEILLANCE CONSIDERATIONS

FOR GAMMA ALGORITHMS $\ldots \ldots \ldots \ldots \ldots \ldots \ldots \ldots$ E-1 


\section{PURPOSE}

This procedure provides a framework for implementation of radiochemical data verification and validation for environmental remediation activities. It has been developed through participation of many individuals currently involved in analytical radiochemistry, radiochemical validation, and validation program development throughout the DOE complex. It should be regarded as a guidance to use in developing an implementable radiochemical validation strategy.

This procedure provides specifications for developing and implementing a radiochemical validation methodology flexible enough to allow evaluation of data useability for project-specific Data Quality Objectives (DQO). Data produced by analytical methods for which this procedure provides limited guidance are classified as "non-routine" radionuclides and methods, and analyses by these methods may necessitate adoption of modified criteria from this procedure.

This procedure is applicable to radionuclide contaminants routinely analyzed by common radioanalytical methods primarily in aqueous and soil/sediment matrices for Environmental Restoration (ER) activities. This procedure is applicable to radionuclide data produced through radioanalytical methods which use instrumentation for detecting activity. This procedure is not applicable to mass spectrometric or fluorimetric methodologies. An example listing of radionuclides and general methodologies to which this procedure applies is provided in Appendix D.

Specifications in this procedure should be incorporated into appropriate project documentation such as the Sampling and Analysis Plan or Project Work Plan and into contractual Statements of Work (SOW) between the project and the analytical laboratories. This procedure shall be used as a baseline used to create project-specific procedures and checklists needed to perform radiochemical data verification and validation.

In this procedure, the word "shall" is used to denote a requirement; the word "should" is used to denote a recommendation; and the word "may" is used to denote permission, neither a requirement nor a recommendation. In conformance to this procedure, all analyses shall be performed in accordance with its requirements, but not necessarily with its recommendations; however, justification must be documented for deviations from recommendations.

\section{REFERENCES}

ANSI N13.30, Performance Criteria for Radiobioassay. 1/15/93.

ANSI N42.14, Calibration and Use of Germanium Spectrometers for the Measurement of Gamma-Ray Emission Rates of Radionuclides. October 30, 1991.

ANSI N42.12-1980, American National Standard Calibration and Usage of Sodium Iodide Detector Systems. April 28, 1980.

ANSI N42.15-1990, American National Standard Performance Verification of Liquid-Scintillation Counting Systems. April 23. 1990. 
ANSI N42.2, Measurement Quality Assurance for Radioassay Laboratories, Rev. 12, Draft. June 1, 1993

Bechtel National, Inc. Review and Verification of QC Level I, II, and III Radiological Data, PP: 1503.2, Rev. 2.

Currie, L.A. "Limits for Qualitative Detection and Quantitative Determination, Application to Radiochemistry." Analytical Chemistry. 40, 3 (1968).

DOE Methods for Evaluating Environmental and Waste Management Samples DOE/EM-0089T, Rev. 2, October, 1994.

FEMP Data Validation Procedure, SSOP-1004. 06/23/93

EG\&G Idaho National Engineering Laboratory. Standard Operating Procedure for Radiological Data Validation. MSO-SOP-12.1.2. January, 1993.

EG\&G Rocky Flats, Environmental Monitoring and Assessment Division. Radiochemical Data Validation Guidelines. May, 1990.

IEEE Standard for Software Verification and Validation Plans. IEEE Std. 1012-1986.

Kanipe, L.G. Handbook for Analytical Quality Control in Radioanalytical Laboratories. EPA-600/7-77-088.

Karnofsky, J. MDAs for Radiochemistry Data Validation, Draft \#2. October, 1994.

Karnofsky, J. Radiochemistry Data Validation, Draft \#4. November, 1993.

Knoll, G. F. Radiation Detection and Measurement, second edition. John Wiley and Sons. 1989

Montgomery. D., and D.E. McCurdy. Specifications for WEMCO Environmental Radioanalytical Services Contract. October 23, 1992.

Science Applications International Corporation. Laboratory Data Validation Guidelines for Evaluating Radionuclide Analyses. Rev. 05A.

Taylor, J.K. Quality Assurance of Chemical Measurements. Lewis Publishers, 1987.

United States Environmental Protection Agency. Manual for the Certification of Laboratories Analyzing Drinking Water, Criteria and Procedures Quality Assurance, Third Edition. EPA/570/9-90/008.

United States Environmental Protection Agency. Data Quality Objectives Process for Superfund. EPA/540/G-93/071

United States Environmental Protection Agency. Guidance for the Data Quality Objectives Process. EPA QA/G-4. August, 1994. 
United States Environmental Protection Agency. Prescribed Procedures for Measurement of Radioactivity in Drinking Water. EPA-600/4-80-032.

United States Environmental Protection Agency. Region IV Hazardous Waste Site Field Sampling Workshop. June 1993.

United States Environmental Protection Agency. "Radiochemical Determination of Gross Alpha and Gross Beta Particle Activity in Water," Radiochemical Procedures Manual EPA-520/5-84-006.

United States Environmental Protection Agency. Upgrading environmental Radiation Data. Health Physics Society Committee Report HPSR-1 (1980). EPA 520/1-80-012.

\section{DEFINITIONS}

Affected Sample Result: a sample result that is considered to be significantly influenced by a quality deficiency, and is qualified, accordingly, through analytical data validation.

Analytical Batch: An analytical batch is a group of sample aliquots analyzed together on the same instrument detector system.

Analytical Data Validation: a technically based analyte and sample specific process that extends the qualification process beyond method or contractual compliance and provides level of confidence in the data that an analyte is present or absent and if present, the associated variability. Data validation is a systematic process, performed external from the data generator, which applies a defined set of performance-based criteria to a body of data that may result in physical qualification of the data. Data validation occurs prior to drawing a conclusion from the body of data.

Analytical Data Verification: a process of evaluating the completeness, correctness, consistency, and compliance of a set of facts against a standard or contract. Data verification is defined as a systematic process, performed by either the data generator or by an entity external to the data generator.

Calibration Verification: Calibration verification, as described in this procedure, is defined as a periodic evaluation of instrument standardization established during initial calibration. Using tolerance or statistical control charts, calibration verification can alert the instrument user of the occurrence of out-of-control instrumental conditions.

Carrier: A carrier is a stable element/compound, introduced into the sample preparation/analysis process, that will behave chemically similar to the analyte isotope(s). It is by virtue of this chemical similarity that the carrier will "carry" the analyte isotope(s) through the sample preparation/analysis process. The amount of the carrier recovered at the end of the analysis compared to that added initially is often used in the calculation of the final result.

Correctable Problem: Correctable problems are deficiencies within data packages which may be rectified through consultation with the laboratory. Correctable problems may be revealed during both data verification and data validation. 
- correctable problems revealed during verification are those deficiencies that can be addressed by obtaining additional information from the laboratory

- correctable problems revealed during validation are those deficiencies with analyses that can be solved by either a second preparation and/or analysis of a sample.

Counting uncertainty: Counting uncertainty, as described in this procedure, is defined as the statistical sample standard deviation, which is an approximation of the population standard deviation, and is numerically defined as the square root of the number of counts obtained from a detector. This relationship holds true, provided that the distribution that the counts follows the Poisson distribution. Units for counting uncertainty are the same as for the reported result and the MDC.

Initial Calibration: Initial calibration, as described in this procedure, is defined as the standardization of an instrument used in radioactivity detection against a traceable radioactive source(s) of known identity and quantity. This standardization prevails until such time as analytical conditions are deemed out of acceptable tolerance or statistical control limits.

Holding Time: Holding time, as described in this procedure, is defined as the period of time between sample collection and sample activity detection.

Minimum Detectable Activity (MDA) : The amount of a radionuclide, which if present in a sample, would be detected with a $\beta$ probability of non-detection while accepting a probability, $\alpha$, of erroneously detecting that radionuclide in a appropriate blank sample. For this procedure, the $\alpha$ and $\beta$ probabilities are both set at 0.05 .

Minimum Detectable Concentration $(M D C)$ : The MDA expressed in concentration units relative to the sample weight or volume.

Non-correctable problem: Non-correctable problems are those deficiencies, within data packages that cannot be addressed through additional laboratory submittals, and sample results must stand as-is.

Non-Correctable Problem: Non-correctable problems are deficiencies within data packages which preclude the evaluation of data quality by predefined criteria. Non-correctable problems may be revealed during both data verification and data validation.

Preparation Batch: A preparation batch is a group of sample aliquots prepared together at the same time using the same method and related to the same quality-indicator samples.

Quality Control Chart: For purposes of this procedure, a quality control chart is used to determine if the response of the instrument has changed statistically; the magnitude the statistical response change may or may not be significant when compared to the required precision and accuracy criteria for the overall analytical technique.

Quality-indicator Sample: Quality-indicator samples are those samples made ready in the laboratory which provide direct or indirect evaluation of the status of analytical system and resulting data quality. Collectively, quality indicator samples are the laboratory control sample, laboratory duplicate, matrix spike, and method blank. 
Reporting Batch: A reporting batch is a group of sample results reported together in a single data package. The reporting batch may be comprised of samples prepared and analyzed together in the same preparation batch or samples prepared and analyzed in different preparation or analytical batches.

Required Detection Limit $(R D L)$ : The RDL is a contractually specified detection limit (MDA or MDC) which, under typical analytical circumstances, should be achievable.

Standard Reference Material (SRM): A material or substance of one or more properties of which are sufficiently well established to be used for the calibration of an apparatus, the assessment of a measurement method, or for assigning values to materials. The SRM is characterized by the U.S. National Institute of Standards and Technology (NIST) or other certified testing authority, and issued with a certificate providing the results of the characterization.

Tolerance Chart: For purposes of this procedure, a tolerance chart is based upon maintaining a change of instrument response to a tolerance level judged acceptable to meet overall quality requirements for the technique; a tolerance level should never be more restrictive than what is statistically possible.

Total Propagated Uncertainty (TPU): The addition of the square root of the sum of the squares of random components of the individual uncertainties, plus the magnitude of the estimated individual systematic relative uncertainties. TPU may include uncertainties introduced through field sampling and analytical laboratory procedures. For the purposes of this procedure, TPU includes only those random and systematic uncertainties associated only with laboratory preparation and analysis. Refer to Appendix $\mathrm{C}$ for a full description of TPU.

$$
T P U=\sqrt{\Sigma R_{i}^{2}+\Sigma s_{j}^{2}}
$$

$\mathrm{R}=$ rand om components of individual relative uncertainties

$\mathrm{s}=$ magnitude of the estimated individual systematic relative uncertainties

Traceable Reference Material (TRM): A NIST prepared standard reference material or a sample of known activity or concentration prepared from a NIST standard reference material (derived standard material).

Tracer: A tracer is a radioactive isotope, introduced into the sample preparation/analysis process, that will behave chemically similar to the analyte isotope(s). The tracer isotope is of the same element as the analyte isotope(s) except where the decay mode, half-life, or availability dictate the use of the isotope of a different element. The activity of tracer detected at the end of the analysis compared to that added initially is used in the calculation of the final result.

Turn-around Time: Turn-around time is contractually specified as the amount of time which elapses between laboratory receipt of the raw samples and subsequent data receipt by the client. 
Well Characterized Reference Material (WCRM): The WCRM may be derived from a field sample which has been well characterized through multiple analyses providing a high level of confidence of the activity level in the sample. The WCRM may be submitted to NIST for characterization and classification as a TRM.

\section{GENERAL INFORMATION}

\section{A. Relation to Laboratory Statement of Work}

Implementation of this procedure depends on the agreement of work to be performed by analytical laboratory in the form of a project-specific laboratory Statement of Work (SOW). While it is outside the scope of this procedure to provide requirements for the radioanalytical laboratory, validation will be expedited if laboratory deliverables are consistent with the evaluation requirements of this procedure.

B. Reporting Recommendations

All samples should be reported with a background-subtracted sample result. The counting uncertainty, TPU, and MDC must be reported at the same level of confidence (e.g. $\pm 2 s$ at 95\%).

\section{Qualification of Data}

When qualification conditions are met, a qualifier and reason code shall be physically or electronically associated with the affected result(s). If more than one reason code is applied, each must be separated by commas. The listing of available codes is presented in Appendix B. If data are not qualified, a character (e.g. an equals sign, or other alphanumeric symbol different from a qualifier) is entered on the sample result line. As directed or as appropriate, reason codes may be applied without qualifiers.

\section{Problem Identification}

Problems identified through data verification and validation are separated into two categories: correctable problems and non-correctable problems.

1. Correctable problems

- The first category contains those problems which may be rectified through consultation with the laboratory.

- The second subcategory contains those problems are those that can be rectified by either a second preparation and/or analysis of a sample.

2. Non-Correctable Problem: Non-correctable problems are deficiencies within data packages which preclude the evaluation of data quality by predefined criteria. 


\section{E. Data Verification}

Data verification is defined as a systematic process, performed by either the data generator or by an entity external to the data generator, which results in evaluation of the completeness, correctness, consistency, and compliance of a data set against a standard or contract.

If verification is performed by the data generator, a project-level surveillance must be established by which the performance of the verification process is evaluated.

Data verification, at the project level, may be conducted either by the data verifier or by the data validator, whichever expedites the review process. If verification is conducted independently of the validator, it includes two activities. The first activity entails inventory of the data package to ensure compliance with the contract and statement of work, in terms of the required deliverables. The second activity entails to inform the validator that a non-correctable problem has occurred and that data may need qualification. If verification is conducted by the validator, the first activity is conducted similarly, but the second activity may result in prompt qualification of data.

Data verification should provide a mechanism for problem resolution with the laboratory; it should not be an after-the-fact identification of non-correctable deficiencies.

\section{Verification Report}

A verification report is written by the data verifier and takes, as input, the steps in this procedure that are listed as "Verification." The data verifier does not qualify data if verification criteria are not met but indicates in the verification report the circumstances surrounding correctable and non-correctable problems in the data package which will be transferred to the validator for possible qualification. The verification report must be in a standard format, and must remain a part of the analytical data package. An example of a verification report is included in Appendix F.

\section{F. Analytical Data Validation}

Analytical data validation, including field and laboratory data review, is defined as a systematic process, performed external from the data generator, which applies a defined set of performance-based criteria to a body of data which may result in qualification of the data. Data validation is not performed by the analytical laboratory. Data validation provides a level of assurance, based on a technical evaluation, that an analyte is present or absent, and if present, the level of uncertainty associated with the measurement, and occurs prior to drawing a conclusion from a body of data. Analytical data validation for radiochemistry includes a technical review of a laboratory data package covering the evaluation of quality-indicator samples, the identification and quantitation of analytes, and the effect of deficiencies in quality control on analytical sample data.

\section{Validation Report}

A validation report that includes the results of validation activities must be completed by the validator and takes, as input, the verification report and the steps in this procedure that are listed as "Validation." A validation report must be completed for every reporting batch that passes through validation. To expedite writing the validation report, comments must include 
explanations for qualification only if the reason codes do not adequately describe justification for qualification. Comments on data qualification for which reason codes adequately describe qualification reason are not necessary.

Both the verification and validation reports must be completed regardless of who performs the reviews.

\section{PROCEDURE}

The steps in this section describe the processes of radiochemical data verification and validation.

Refer to Appendix A for qualifier and reason code descriptions. Refer to Appendix B for guidance on qualification due to multiple quality deficiencies.

A. Custody of Samples and Sample Documentation

While verification/validation cannot assure a sample has truly been in custody from the field to the final result, an evaluation of field and laboratory chains of custody will provide the best indicator. A sample is defined as being in custody if any of the following conditions are met:

- it is within one's possession;

- it is within one's view, after being in one's possession;

- it was in one's possession and then was secured to prevent tampering;

- it is placed in a designated secure area.

\section{Verification}

Custody of the samples reported in the data package should be verified by tracing the signature record on both field and laboratory chains of custody. Verify that all samples on the chains of custody are present in the reporting batch. If there is a break in custody of the sample(s), representativeness of the samples may be in question. Indicate this problem in the verification report.

Verification of sample documentation includes result report header checks for accuracy from the COC. If header information is incorrect when compared to the COC, the verifier shall mark through the incorrect header entry and pen in the correct entry, placing initials and date next to the correction. If sample identity is in question, every attempt should be made to verify the true identity of each sample, else sample representativeness is in doubt.

\section{Validation}

If sample origin and identity can not be substantiated, sample data may be qualified "R."

\section{B. Holding Time and Turn-around Time}

Holding time is defined for radiochemical data verification and validation as the period of time between sample collection and sample activity detection. Samples must be analyzed within a holding time precluding significant decay of short-lived target radionuclides. Holding 
time should be contractually specified to the laboratory. If holding times are not contractually satisfied, and if other technical corrective actions, such as increased count times or increased sample aliquot volumes or weights, have not been performed to compensate for long holding times, , the RDL may be exceeded

Physical characteristics and matrix influences must also be considered when setting holding times. These characteristics must be considered when planning for validation implementation. The following table presents commonly analyzed radionuclides on the DOE Complex that have characteristics affecting holding time establishment decisions:

Table 5.1. Physical and matrix-related characteristics

\begin{tabular}{|l|l|}
\hline Nuclide & Physical or Matrix-related Characteristic \\
\hline Anionic & Volatile when placed in acid solution \\
\hline${ }^{3} \mathrm{H}$ & Volatile \\
\hline${ }^{99} \mathrm{Tc}$ & Volatile \\
\hline
\end{tabular}

The following table presents commonly analyzed radionuclides on the DOE Complex particularly susceptible to holding time and RDL exceedences due to short half-life:

Table 5.2. Commonly analyzed short half-life radionuclides

\begin{tabular}{|l|l|}
\hline \multicolumn{1}{|c|}{ Nuclide } & \multicolumn{1}{|c|}{$\mathrm{T}_{1 / 3}$} \\
\hline${ }^{131} \mathrm{I}$ & 8.04 days \\
\hline${ }^{222} \mathrm{Rn}$ & 3.82 days \\
\hline${ }^{210} \mathrm{Po}$ & 138 days \\
\hline${ }^{89} \mathrm{Sr}$ & 50.5 days \\
\hline
\end{tabular}

\section{Verification}

If the sample MDC is reported at a level greater than the RDL when the sample result is less than the $\mathrm{MDC}$ or $\pm 2 \mathrm{~s}$ counting uncertainty, and no justification is provided for not reanalyzing at a longer count time or greater sample aliquot, data are noncompliant with the contractual RDL. Note the occurrence of RDL exceedence in the verification report.

Turn-around time is evaluated as strictly a contractual issue. An agreement should be established between the client and the laboratory concerning turn-around times for reported data. If turn-around times are not satisfied, a contractual mechanism should provide for appropriate action.

\section{Validation}

It should be realized that RDL exceedence may occur for reasons other than excessive radionuclide disintegration. Reference should be made to other potential factors such as count time and aliquot size to apply the appropriate reason code(s). The affected results are not 
qualified, but are flagged only with the reason code "Q10" if reanalysis can not be performed or deemed by the project as unnecessary.

C. Sample Preservation

When appropriate, or in the absence of known preservation techniques, the preservatives and container types listed in the following table should be used for aqueous samples.

Table 5.3. Preservation and container requirements

\begin{tabular}{|l|l|l|}
\hline Nuclide or Group & Preservative & Container \\
\hline Gross Alpha or Beta & Concentrated $\mathrm{HCl}$ or $\mathrm{HNO}_{3}$ to $\mathrm{pH} \leq 2$ & Plastic or Glass \\
\hline${ }^{226} \mathrm{Ra}$ or ${ }^{228} \mathrm{Ra}$ & Concentrated $\mathrm{HCl}$ or $\mathrm{HNO}_{3}$ to $\mathrm{pH} \leq 2$ & Plastic or Glass \\
\hline $\mathrm{U}, \mathrm{Th}$ & Concentrated $\mathrm{HCl}$ or $\mathrm{HNO}_{3}$ to $\mathrm{pH} \leq 2$ & Plastic or Glass \\
\hline${ }^{89} \mathrm{Sr} /{ }^{90} \mathrm{Sr}$ & Concentrated $\mathrm{HCl}$ or $\mathrm{HNO}_{3}$ to $\mathrm{pH} \leq 2$ & Plastic or Glass \\
\hline${ }^{131} \mathrm{I}$ & $\mathrm{HCl} / 2 \mathrm{M} \mathrm{NaHSO}$ & Plastic or Glass \\
\hline${ }^{3} \mathrm{H}$ & None & Glass \\
\hline${ }^{99} \mathrm{Tc}$ & Concentrated $\mathrm{HCl}$ or $\mathrm{HNO}_{3}$ to $\mathrm{pH} \leq 2$ & Plastic or Glass \\
\hline Gamma Emitters & Concentrated $\mathrm{HCl}$ or $\mathrm{HNO}_{3}$ to $\mathrm{pH} \mathrm{2}$ & Plastic or Glass \\
\hline
\end{tabular}

United States Environmental Protection Agency. Manual for the Certification of Laboratories Analyzing Drinking Water, Criteria and Procedures Quality Assurance, Third Edition. EPA/570/9-90/008.

1. Verification

If the proper preservative and/or container type have not been used, note the problem in the verification report.

\section{Validation}

If samples have not been preserved properly in the field or have been stored in an improper container, qualify those sample results $<$ MDC "UJ." Sample results $\geq M D C$ may not require qualification; however " $\mathrm{J}$ " may be placed if necessary.

If samples with radionuclides amenable to preservative with acid have not been acidified in the field, but have been acidified in the laboratory prior to subsampling, qualification may not be necessary. The matrix and container type will not affect the radioactive characteristics of the radionuclides in the sample. For this reason, neglecting to acidify samples prior to shipment to a laboratory should not necessarily result in qualification. However, as radionuclides will adhere to the container walls, acidification (of aqueous samples) either during the sampling event or at the laboratory prior to subsampling is critical to ensure that all radioactive components are in solution, and the representativeness of the sample is maintained. 
D. Instrument Calibration

1. Scope

It is outside the scope of this document to prescribe calibration requirements for the laboratory. This section provides recommended frequencies, performance and evaluation criteria, based on existing ANSI standards. Decisions regarding the deliverable requirements for calibration data must be made during the project planning phase and communicated to the laboratory in the SOW. Decisions regarding calibration evaluation strategy should be influenced by the strategy outlined in this section in order to provide a consistent approach to data evaluation with respect to calibration and to expedite the verification and validation processes.

\section{Mechanism for Initial Calibration Evaluation}

All calibration data are essential for project records from the standpoint of defending the conditions under which samples were analyzed; however, evaluation of initial calibration data should not involve verification and validation with each reporting batch.

To provide a mechanism for initial calibration evaluation at the project-level, the following approach should be taken.

a. Laboratory Statements of Work must incorporate calibration definitions, frequency, and quality control criteria.

b. Upon award of a contract, the laboratory must provide to the project all initial calibration data for all detector systems to be used under the contract. At this time, the control status of initial calibration can be evaluated. The contract must require that the laboratory update the project with changes in calibration status. Tracking numbers for the initial calibration must be included in all reporting batches for reference purposes.

c. If the necessary deliverables for initial calibration cannot be provided by the laboratory, two potential options exist:

i. if missing deliverables do not significantly affect the ability to evaluate sample data, the project may accept the initial calibration data

ii. if missing deliverables either present a significant nonconformance for project records retention or preclude the ability to evaluate sample data, the project may request the laboratory to perform a new initial calibration in accordance with the deliverables in this section.

3. General Technical Specifications for Initial Calibration

The following technical specifications apply to all initial calibrations and calibration verifications, independent of counting instrumentation category. 


\section{a. Check Source Statistics}

The instrument calibration sources should provide adequate counting statistics (less than $1 \%$ Poisson statistic uncertainty) over the time period for which the source is to be counted. However, the source shall not be so radioactive as to cause 1) pulse pileups, 2) dead time that is significantly different from that to be expected from routine analysis, or 3 ) gain shift in the case of pulse height analyzer systems.

\section{b. Radioactive Sources}

Commercially prepared and sealed standards shall not be used after their stated expiration dates, which are based on radionuclide half-life or physical form of the standard, e.g. sealed source or plated planchet. Standards prepared at the laboratory or those purchased without expiration dates should be replaced yearly.

The standard source(s) used in initial calibration shall be NIST-traceable Standard Reference Materials, or equivalent; however, source(s) used in calibration verification are not required to be NIST-traceable, unless measurements of these sources are directly used in calculation of analytical sample data results.

\section{c. Control Criteria}

The scope of this document does not include prescriptive requirements for calibration, however, quality of analytical data is highly dependent on control of the calibration process. To facilitate a framework for defining control of the calibration process, the three following strategies may be incorporated dependant on what instrumentation is being used:

i. Tolerance charts may be established based on consideration of specific performance characteristics of the instrument and complexity of the matrix of samples that will be analyzed. The required precision of tolerance charts must never be more restrictive than that of a quality control chart.

ii. Statistical quality control charts may be established based only on a level of confidence considered necessary for statistical quality control.

iii. Fixed limits may be used by consideration of percent deviation from a known value. With some radiochemical methodologies (e.g. alpha and gamma spectroscopy) establishment of tolerance or statistical quality control charts may provide unrealistic precision goals (e.g. 5\% RPD may exceed a \pm 3 s control chart limit but still provide adequate instrumental precision). In these cases evaluation of measured values using a percent deviation approach may provide realistic evaluation of detector precision.

\section{d. Establishment of control points}

Establishment of control points for use with a tolerance or statistical quality control chart may be approached in two differing strategies, fixed range or moving range.

i. Fixed range control charts are established by acquiring a predetermined number of points, with associated mean and standard deviation, and comparing subsequent 
data point acquisitions to those statistical descriptors. This allows for evaluation of instrumental control over time, but may not represent true precision over time.

ii. Moving range control charts are established by acquiring a predetermined number of points, with associated mean and standard deviation, and as subsequent points are acquired, they are included for an up-to-date evaluation of system precision. In using a moving range control chart, only the most recent 20 points are considered in establishing statistical descriptors. The use of moving range control charts allows for real-time evaluation of detector control, but does not allow for evaluation of detector control in relation to initial calibration.

\section{e. Control of Background}

The control limits for check sources and backgrounds (where applicable) shall be established using a minimum of 20 sequentially measured data points. . For extended background count periods, a series of at least 10 single background measurements is acceptable. No samples, subject to these specifications may be counted until these warning and control limits have been established.

Background count time should be equal to or greater than sample count time unless precluded by extended low level sample count times, in which case background count time may be less than sample count time.

\section{f. Geometry}

With all methods of detection, the calibration counting geometry used should be the same as that used with the analytical samples.

\section{g. Background subtraction}

Calibration data should be background-subtracted, whether data is used in generation of efficiencies, cross-talk, or resolution evaluation.

\section{h. Recalibration}

Recalibration should only be necessary in the event that the instrument/system has malfunctioned and the repaired equipment has responded to a QC test in a fashion that the tolerance level of a control chart has been exceeded, i.e. the operating or response characteristics of the instrument/system have changed more than the tolerance/control limits permit. Detector calibration is verified according to frequencies that will satisfy contractual criteria, and according to criteria defining the warrant of corrective action.

\section{Specific Technical Specifications for Calibration Verification}

a. Calibration verification data must be submitted with each reporting batch and will be evaluated at that frequency. If samples within a reporting batch are from separate counting batches, calibration verification documentation must be included for all counting batches. 
b. Each reporting batch submitted from the laboratory to the project should contain control charting data related to calibration verification for all detectors used in the analyses of the analytical samples.

c. Calibration verification is performed and monitored with tolerance or QC charts for instrumental parameters specific to each type of detector. If the daily check source count result exceeds the tolerance limits or $\pm 3 \sigma$ control limit of the appropriate 20 points, the laboratory should recount the check source to verify the out of control condition. If the recount again exceeds the control limit, the system is considered out of control, and no samples shall be run on that system until it is brought back into control. If the recount is in control a third count shall be done and if in control, analytical sample counting may continue, otherwise no samples shall be analyzed on that system until it is brought back into control. Any samples counted after the last in-control check standard must be recounted except for those where decay has eliminated that radionuclide.

d. If calibration verification data exceed the tolerance limits or the $\pm 3 \sigma$ control limits, reference must be made to quality control sample data in the data package to determine the extent of calibration nonconformance on the analytical batch. Exceedence of control limits may not constitute qualification of data; but conversely, excessive control limit exceedence may affect all data in an analytical batch, justifying qualification.

5. Initial Calibration for Gross Alpha and Gross Beta by Gas Proportional Systems

Initial calibration review consists of evaluations of efficiency, background, and cross talk.

a. Deliverables

The basic deliverables required to complete the checks are described in this section:

i. Summary report(s) for both gross alpha and gross beta including the following:

- dates of calibration and planchet preparation;

- mass attenuation curves including mass of salts, planchet diameter, efficiencies at each mass, and mathematical relationships developed from self absorption curves;

- matrix residue identity used for curve establishment - e.g. ASTM Type II water;

- identities of nuclides used for calibration;

- geometries;

- backgrounds;

- charts of voltage plateaus;

- number of counts accumulated in each channel for each standard;

- count times for all analyses;

- calculated activities for all analyses;

- cross talk factors

ii. Evidence of decay correction of standards prior to calculation of efficiencies, if appropriate;

iii. All raw data supporting initial calibration; 
iv. Certificates for standards used in calibration.

\section{b. Frequency}

An initial calibration for gas proportional counters should be performed when out-of-control conditions, indicated from control charting, necessitate recalibration of the instrument or if the project requests recalibration based on deliverable deficiencies.

\section{c. Performance Criteria}

The following criteria are recommended in the establishment of initial calibration mass attenuation curves for gas proportional counters:

i. Each alpha and beta calibration standard shall be counted to an accumulation of 10,000 counts

ii. Each mass attenuation curve should consist of at least 10 points well distributed throughout the mass range unless instrument specific programs designate otherwise

iii. Operating voltage on the plateau shall be established to achieve a cross talk of $\alpha$ into the $\beta$ channel of $<10 \%$, and $\beta$ into the $\alpha$ channel of $<1 \%$

iv. The instrument background must be checked at the time of initially calibrating the detector.

v. The matrix residue used in establishment of the mass attenuation curves must be reasonably well matched to the expected sample matrix.

vi: Plated planchets used must be less than 3 years old

vii. The maximum calibration planchet density for alpha and beta counting should be $\leq 5 \mathrm{mg} / \mathrm{cm}^{2}$.

viii. The sources used for the determination of cross talk should be of similar geometry and isotope content to that of the analytical samples; however, when performing analyses for gross measurement, a standard isotope source is acceptable (e.g. ${ }^{241} \mathrm{Am}$ for gross alpha, and ${ }^{137} \mathrm{Cs},{ }^{90} \mathrm{Sr}{ }^{90} \mathrm{Y}$ for beta calibration).

ix. Standard activity shall be decay corrected (if applicable) prior to calculation of instrument efficiency.

6. Calibration Verification for Gross Alpha and Gross Beta by Gas Proportional Systems

Calibration verification consists of acceptable efficiency and background data

a. Deliverables

The basic deliverables required to complete the checks are described in this section:

i. Matrix residue identity used for check source; 
ii. Date of preparation of planchets used in calibration verification;

iii. Number of counts in each channel for each mass-efficiency calibration standard;

iv. Calibration points including mass on planchet and attenuation factor;

v. Tolerance chart or statistical control chart of the the appropriate 20 efficiencies with $\pm 3 \sigma$ limits with residue weights for each efficiency verification;

vi. Tolerance chart or statistical control chart of the appropriate 20 instrument backgrounds with $\pm 3 \sigma$ limits;

vii. Listing of $\mathrm{X} / \mathrm{Y}$ coordinates used in constructing the control charts;

viii. Evidence of decay correction of standards prior to calculation of efficiencies, if appropriate;

ix. Geometries used in analysis.

b. Frequency

i. Calibration verification must occur on a daily basis, or prior to use. The only exception to this specification is when performing low level counts with extended count times precluding the verification of calibration on a daily basis. In this case, calibration may be verified on a weekly basis.

ii. Both the operating voltage (on the plateau) and the cross talk shall be checked on a semi-annual basis.

c. Performance Criteria

i. Each alpha and beta verification standard shall be counted to an excess of 10,000 counts

ii. The check source count result for both alpha and beta should be maintained on a control chart with the mean and $\pm 3 \sigma$ limits plotted. If the daily check source count result, for either alpha or beta, exceeds the tolerance or statistical $3 \sigma$ control limit, the checks source must be recounted to verify the measurement was correct. If the recount again exceeds the control limit, the system is considered out of control, no samples shall be run on that system until it is brought back into control. If the recount is in control a third count shall be done and, if in control, analytical sample counting may continue, otherwise no samples shall be run on that system until it is brought back into control. Any samples counted after the last in-control check standard shall be recounted.

iii. Efficiency for both alpha and beta must remain within tolerance or statistical $\pm 3 \sigma$ control limits of the appropriate 20 efficiencies.

iv. Instrument background for both alpha and beta must be maintained on a control chart with the mean and $\pm 3 \sigma$ limits plotted. 
v. Following gas changes, gas should be allowed to flow for a period of time necessary to purge the system; check source and background counts must be performed following a gas change

\section{d. Verification}

Verification of completeness of deliverables must be performed prior to validation. If deliverables are found to be inconsistent with the listing in this section, contact the laboratory to request the additional information. If the information can not be obtained, indicate this in the verification report as a non-correctable problem

\section{e. Validation}

If the frequency of calibration verification does not meet the frequency specifications, place the appropriate reason code on the affected data. Control charts shall be evaluated for out-of-control conditions. If any of these conditions are met, reference must be made to the laboratory case narrative for justification for analyzing analytical samples under non-compliant conditions; QC samples should be evaluated to determine if the non-compliant check source is indicative of a systemic problem or if it is a chance occurrence. If the occurrence is considered a chance occurrence, and samples do not seem affected, no qualification action is necessary. If the occurrence is considered systemic, and sample results seem to be affected, sample results may be qualified "J" or " $R$ " based on specific conditions in the analytical batch.

If standard source(s) have aged greater than the expiration date on the certificate(s), affected sample results should be qualified "J," and qualified " $\mathrm{R}$ " using professional judgement.

\section{Initial Calibration for Liquid Scintillation}

Initial calibration review consists of acceptable efficiency and background data

\section{a. Deliverables}

The basic deliverables required to complete the checks are described in this section:

i. Summary report(s) including dates of calibration, geometries, efficiency, background, quench levels, count times for all analyses, number of counts accumulated for each standard, measured activities for all analyses;

ii. QC source identity;

iii. Matrix used for efficiency curve establishment (e.g. ASTM Type II water);

iv. Evidence of decay correction of standards prior to calculation of efficiencies, if appropriate;

v. Calibration points including quench level and measured results;

vi. All raw data supporting initial calibration; 
vii. Certificates for standards used in calibration.

b. Frequency

An initial calibration for liquid scintillation systems should occur when out-of-control conditions, indicated from control charting, necessitate recalibration of the instrument or if the project requests recalibration based on deliverable deficiencies.

\section{c. Performance Criteria}

i. The quench curve standard's vial characteristics (glass or plastic) and volume shall be similar to that of the samples to be counted.

ii. The efficiency standard shall be counted prior to each analytical batch and shall be counted to a low counting error (less than $1 \%$ at $3 \sigma$, or 100,000 counts) at least twice. The average of the efficiencies shall be used to calculate the activity of the samples. The standard need not be prepared daily, but shall be replaced if a decrease in efficiency is noted or phase separation is apparent in the cocktail.

iii. The tritiated water solution prepared from the flame-sealed NIST standard reference material (or equivalent) shall be replaced or recalibrated every 6 months with a fresh standard.

iv. For those labs using the internal standard method of quench correction, the tritium standard used to spike the samples shall be recalibrated or replaced on an annual basis.

v. For those labs using an external quench monitor and a variable quench protocol, a minimum of ten standards, with quench's well distributed over the appropriate range, shall be used to determine the quench curve. Each standard in the quench set will be counted to accumulate a minimum of 100,000 counts.

\section{Calibration Verification for Liquid Scintillation}

Calibration verification consists of acceptable efficiency and background data.

a. Deliverables

The basic deliverables required to complete the checks are described in this section:

i. Matrix used for check source (e.g. ASTM Type II water);

ii. Calibration point(s) including quench level, number of counts for each point, and measured result(s);

iii. Tolerance chart or statistical control chart of the appropriate 20 efficiencies with $\pm 3 \sigma$ limits

iv. Tolerance chart or statistical control chart of the appropriate 20 instrument backgrounds with $\pm 3 \sigma$ limits; 
v. Listing of $\mathrm{X} / \mathrm{Y}$ coordinates used in constructing the control charts;

vi. Geometries used in analysis;

vii. Evidence of decay correction of standards prior to calculation of efficiencies, if appropriate.

\section{b. Frequency}

Calibration verification must occur on a daily basis, or prior to use. The only exception to this specification is when performing low level counts with extended count times precluding the verification of calibration on a daily basis. In this case, calibration may be verified on a weekly basis.

\section{c. Performance Criteria}

i. On each day that samples will be counted, an unquenched flame-sealed check source vial, shall be counted prior to sample counting. In excess of 10,000 counts of the respective activity shall be accumulated.

ii. On each day that samples will be counted, the background count rate shall be determined by counting a vial free of the analyte(s) of interest. The duration of this background count shall be as long, if not longer, than that for the analytical samples. This determination shall be made separate from any vials that are counted to meet method blank requirements of the respective statement of work.

iii. For those labs that quench correct the background activity, each day that samples will be counted, a vial of the quenched background shall be counted to ensure control of the instrument background. The quench level shall lie within the tolerance limits of the set of 20 used in establishing initial calibration.

iv. For tritium analyses, the laboratory shall show that the water used for the background, to be free of tritium activity (by comparison to an EPA blank water or through other means).

v. If the daily check source count result or the background count rate result exceeds the 3 sigma limit, recount to verify the out of control condition. If the recount again exceeds the control limit, the system is out of control, no samples shall be run on that system until it is brought back into control. If the recount is in control a third count shall be done and if in control analytical sample counting may continue, otherwise no samples shall be analyzed on that system until it is brought into control. Any samples analyzed after the last in-control check standard shall be reanalyzed.

\section{d. Verification}

Verification of completeness of deliverables must be performed prior to validation. If deliverables are found to be inconsistent with the listing in this section, contact the laboratory to request the additional information. If the information can not be obtained, indicate this in the verification report. 
e. Validation

If the frequency of calibration verification does not meet the frequency specifications, place the appropriate reason code on the affected data. Control charts shall be evaluated for of out-of-control conditions. If any of these conditions are met, reference must be made to the laboratory case narrative for justification for analyzing analytical samples under non-compliant conditions; QC samples should be evaluated to determine if the non-compliant check source is indicative of a systemic problem or if it is a chance occurrence. If the occurrence is considered a chance occurrence, and samples do not seem affected, no qualification action is necessary. If the occurrence is considered systemic, and sample results seem to be affected, sample results may be qualified "J" or " $R$ " based on specific conditions in the analytical batch.

If standard source(s) have aged greater than the expiration date on the certificate(s), affected sample results should be qualified "J," and qualified " $\mathrm{R}$ " using professional judgement.

\section{Initial Calibration for Alpha Spectrometry}

Special note for alpha spectrometry calibration. Where a method or Statement of Work do not require the determination of tracer recovery, the determination of detector counting efficiency is not necessary. In this circumstance, the following specifications for efficiency calibration are not applicable.

Initial calibration review consists of acceptable efficiency, background, and peak resolution data.

a. Deliverables

The basic deliverables required to complete the checks are described in this section:

i. Summary report(s) including dates of calibration, efficiency, background, peak(s) resolution, count times for all analyses, number of counts accumulated in each channel for each standard, measured activities for all analyses;

ii. Matrix used for efficiency establishment (e.g. ASTM Type II water);

iii. Evidence of decay correction of standards prior to calculation of efficiencies, if appropriate;

iv. Calibration points including efficiency, energy, and peak resolution for relevant peaks;

v. All raw data supporting initial calibration;

vi. Certificates for standards used in calibration; 
b. Frequency

An initial calibration for alpha spectrometry systems should occur when out-of-control conditions, indicated from control charting, necessitate recalibration of the instrument or if the project requests recalibration based on deliverable deficiencies.

c. Performance Criteria

i. A single check source may be used for efficiency calibrations, and should consist of ${ }^{241} \mathrm{Am},{ }^{238} \mathrm{Pu},{ }^{239} \mathrm{Pu}$, or ${ }^{244} \mathrm{Cm}$. A running average of up to 5 efficiencies for one or more of these radionuclides may be used as the efficiency for sample calculations.

ii. At least two, preferably three, isotopes shall be used for energy calibrations. The isotopes used should be from those listed above.

iii. Radionuclides should be selected which have energies that will limit the detection of attenuated alpha events of higher energies. The sources should be of a high enough quality as to limit the amount of tailing created by attenuation. Peaks outside the ROI need not be identified.

10. Calibration Verification for Alpha Spectrometry

Calibration verification consists of acceptable efficiency, background, and peak resolution data.

a. Deliverables

The basic deliverables required to complete the checks are described in this section:

i. Tolerance chart or statistical control chart of the appropriate 20 efficiencies, peak energies or channel numbers with $\pm 3 \sigma$ limits;

ii. Background results;

iii. Resolution demonstration of relevant peak(s);

iv. Listing of peak energies;

v. Listing of $\mathrm{X} / \mathrm{Y}$ coordinates used in constructing the control charts;

vi. Evidence of decay correction of standards prior to calculation of efficiencies, if appropriate;

vii. Geometries used in analysis.

b. Frequency

Calibration verification will be performed weekly prior to analytical sample counting, unless analytical conditions warrant more frequent verification. 
The background count rate shall be determined at least once per month by counting a clean mount of the same geometry to sample mounts. The duration of this background count shall be at least 12 hours.

\section{c. Performance Criteria}

i. The check source shall be counted a sufficient period of time to accumulate an excess of 2,000 counts in each region of interest. This check source count will be used to monitor counting efficiency, peak energy, and peak resolution. The region of interest of a minimum of one isotope from the following $\left({ }^{241} \mathrm{Am},{ }^{238} \mathrm{Pu},{ }^{239} \mathrm{Pu}\right.$, or ${ }^{244} \mathrm{Cm}-{ }^{234} \mathrm{U}$ and ${ }^{238} \mathrm{U}$ are also recommended) shall be used to monitor these performance parameters.

ii. If the daily check source count result exceeds the 3 sigma limit, recount to verify the out of control condition. If the recount again exceeds the control limit, the system is out of control, no samples shall be run on that system until it is brought back into control. If the recount is in control a third count shall be done and if in control analytical sample counting may continue, otherwise no samples shall be analyzed on that system until it is brought into control. Any samples analyzed after the last in-control check standard shall be reanalyzed.

iii. The isotope(s) radionuclides/isotopes chosen for evaluation of peak resolution should be the same as used in the initial calibration. The resolution of the detector shall be sufficient to minimize the tailing of counts from peaks of higher energy into regions of interest (ROI) of lower energy peaks.

iv. The background count rate of a detector used for alpha spectrometry will naturally increase in those regions of interest corresponding to radionuclides present in the counted samples. This background count rate is corrected for in the result calculations and thus has little impact on the quality of the reported data. However, it is possible that over time this background count rate could increase to a level where the RDL of some analytes are impacted, unless the lab has corrected for this with longer counting times. If this problem should be observed, the corrective action of cleaning or replacing the detector should be discussed with the laboratory.

\section{d. Verification}

Verification of completeness of deliverables must be performed prior to validation. If deliverables are found to be inconsistent with the listing in this section, contact the laboratory to request the additional information. If the information can not be obtained, indicate this in the verification report.

\section{e. Validation}

If the frequency of calibration verification does not meet the frequency specifications, place the appropriate reason code on the affected data. Control charts shall be evaluated for of out-of-control conditions. If any of these conditions are met, reference must be made to the laboratory case narrative for justification for analyzing analytical samples under non-compliant conditions; QC samples should be evaluated to determine if the non-compliant check source is indicative of a systemic problem or if it is a chance occurrence. If the 
occurrence is considered a chance occurrence, and samples do not seem affected, no qualification action is necessary. If the occurrence is considered systemic, and sample results seem to be affected, sample results may be qualified " $J$ " or " $R$ " based on specific conditions in the analytical batch.

If standard source(s) have aged greater than the expiration date on the certificate(s), affected sample results should be qualified "J," and qualified " $R$ " using professional judgement.

\section{Initial Calibration for Gamma Spectrometry}

Initial calibration review consists of acceptable peak energy, efficiency, background and peak resolution data

\section{a. Deliverables}

The basic deliverables required to complete the checks are described in this section:

i. Summary report(s) including dates of calibration (energy, efficiency, background, peak(s) resolution), geometry, count times for all analyses, number of counts accumulated in each channel for each standard, measured activities for all analyses;

ii. Matrix used in the geometry standard (e.g. epoxy);

iii. Density of standard;

iii. Evidence of decay correction of standards prior to calculation of efficiencies, if appropriate;

iv. Calibration points including efficiency, energy, and peak resolution for relevant peaks;

v. All raw data supporting initial calibration;

vi. Certificates for standards used in calibration.

\section{b. Frequency}

An initial calibration for gamma spectrometry systems should occur when out-of-control conditions, indicated from control charting, necessitate recalibration of the instrument or if the project requests recalibration based on deliverable deficiencies. An initial energy/efficiency calibration for each geometry in conjunction with the preparation of a tolerance or QC chart shall be performed, thus linking the calibration to the tolerance/QC chart.

The energy/efficiency calibration of gamma spectrometers shall be performed at least semi-annually. The energy/efficiency calibration standard shall have at least three gamma emitting energies. If there are only 3 energies in the calibration standard, then the difference between the energies shall be at least $500 \mathrm{keV}$ with one energy being less than $300 \mathrm{keV}$. Energy calibration photopeaks shall have $1332 \mathrm{keV}$ (cobalt-60) Full Width at Half Maximum (FWHM) values of less than $4 \mathrm{keV}$ for $\mathrm{HPGe}$ and $\mathrm{Ge}(\mathrm{Li})$ detectors. Where Nal detectors are 
used for an analysis, as permitted by the method/SOW, the FWHM shall be less than 1.5 times the instrument manufacturer's

stated specification for FWHM.

c. Performance Criteria

i. Where the method is not specific to a single radionuclide using a single energy (e.g. cesium-137 using only $661 \mathrm{keV}$ ), the calibration source shall have several emissions over an energy range of about $0-2000 \mathrm{keV}$.

The source shall not be used for calibration for more than 4 half-lives beyond the calibration date indicated on the certificate of calibration.

ii. The calibration source shall be counted to accumulation of an excess of 20,000 counts in each region of interest.

iii. The energy calibration standard shall have at least three gamma emitting energies. The difference between the energies shall be at least $500 \mathrm{keV}$ with one energy being less than $300 \mathrm{keV}$. Energy calibration photopeaks shall have Full Width at Half Maximum (FWHM) values of less than $4 \mathrm{keV}$.

12. Calibration Verification for Gamma Spectrometry

Calibration verification consists of acceptable peak energy, efficiency, background and peak resolution data.

a. Deliverables

The basic deliverables required to complete the checks are described in this section:

i. Tolerance chart or statistical control chart of the appropriate 20 efficiencies with $\pm 3 \sigma$ limits

ii. Tolerance chart or statistical control chart of the appropriate 20 relevant peak energies with $\pm 3 \sigma$ limits;

iii. Resolution demonstration of relevant peak(s);

iv. Listing of $\mathrm{X} / \mathrm{Y}$ coordinates used in constructing the control charts;

v. Evidence of decay correction of standards prior to calculation of efficiencies, if appropriate;

vi. Geometries used in analysis.

b: Frequency

i. Energy, efficiency and resolution calibration verification must occur on a daily basis or prior to use. The only exception to this specification is when performing low level counts with extended count times precluding the verification of 
calibration on a daily basis. In this case, calibration may be verified on a weekly basis.

ii. The background count rate for the representative geometries shall be determined at least once per week. Two alternatives should be considered in counting the background rate: by counting each representative geometry filled to the respective volume with distilled or deionized water, or counting without a representative geometry, an empty cave. The duration of the background count shall be as long, if not longer, as that of the respective samples.

\section{c. Performance Criteria}

i. The check source used to verify energy calibration should include radionuclides with energies covering the expected energy range of the radionuclides of interest. It is recommended that low, mid, and high energy radionuclides be included for verification of energy, efficiency, and peak resolution.

ii. Energies of the low, mid and high energy radionuclides should fall within $\pm 0.2 \mathrm{keV}$ of the initial calibration energies.

iii. The check source shall be counted to accumulation of an excess of 20,000 counts in the low, mid, and high energy ranges of interest. Examples of checksources which will cover common radionuclide energy ranges are listed in the previous section.

These emissions shall not be used for calibration verification for more than 4 half-lives beyond the calibration date indicated on the certificate of calibration.

iv. If the daily check source count result for counting efficiency at a low, mid, and high points exceeds the tolerance chart or statistical $3 \sigma$ control limit, recount to verify the out of control condition. If the recount again exceeds the control limit, the system is out of control, no samples shall be run on that system until it is brought back into control. If the recount is in control a third count shall be done and if in control sample counting may continue, otherwise no samples shall be run on that system until it is brought back into control. Any samples counted after the last in-control check standard shall be recounted.

v. Peak resolution from the low, mid, and high energy ranges should be $< \pm 1 \mathrm{FWHM}$ from the respective peaks in the initial calibration.

\section{d. Verification}

Verification of completeness of deliverables must be performed prior to validation. If deliverables are found to be inconsistent with the listing in this section, contact the laboratory to request the additional information. If the information can not be obtained, indicate this in the verification report. 


\section{e. Validation}

If the frequency of calibration verification does not meet the frequency specifications, place the appropriate reason code on the affected data. Control charts shall be evaluated for of out-of-control conditions. If any of these conditions are met, reference must be made to the laboratory case narrative for justification for analyzing analytical samples under non-compliant conditions; QC samples should be evaluated to determine if the non-compliant check source is indicative of a systemic problem or if it is a chance occurrence. If the occurrence is considered a chance occurrence, and samples do not seem affected, no qualification action is necessary. If the occurrence is considered systemic, and sample results seem to be affected, sample results may be qualified "J" or "R" based on specific conditions in the analytical batch.

If standard source(s) have aged greater than the expiration date on the certificate(s), affected sample results should be qualified " $J$," and qualified "R" using professional judgement.

E. Quality-indicator samples

Quality-indicator samples are evaluated during data validation to determine the control of the analytical method, and under what conditions the usability of data has been affected.

The strategy by which quality-indicator samples are evaluated involves an evaluation of whether the difference between expected and measured results is statistically significant when compared to their Total Propagated Uncertainties (TPU). The mathematical relationships presented in the following sections are compared to a factor corresponding to a statistical level of confidence. When the relationship exceeds the factor, the two results differ at that statistical level of confidence when compared to their TPU.

The statistical assumption inherent in these tests is that sample results are drawn from normally distributed populations with estimated means and known variances. Factors in the TPU relationship may originate from populations which are not necessarily normally distributed (e.g. counting uncertainty). However, use of sample results and TPU, assuming approximation to the normal distribution, provides a reasonable and appropriate approach to evaluating control of analytical conditions. Presented in this procedure are statistical decision-making levels at $95 \%$ and $99 \%$ levels of confidence (decision-making factors are 1.96 and 2.58, respectively). Projects may choose other levels of confidence and decision-making factors based on project DQOs, with the realization that qualification decisions made through validation will be at differing levels of confidence and conservatism. The following table provides examples of these decision-making factors which are applied as decision-making tools through this procedure: 
Table 5.4 Examples of confidence levels for qualification decision-making

\begin{tabular}{|c|c|c|}
\hline Confidence Level, \% & $\begin{array}{c}\text { Decision-making } \\
\text { factor }\end{array}$ & Decision-making level \\
\hline 50 & 0.68 & more conservative \\
\hline 67 & 1.00 & \multirow{2}{*}{} \\
\hline 75 & 1.15 & \\
\hline 90 & 1.645 & \\
\hline 95 & 1.960 & \multirow{2}{*}{ less conservative } \\
\hline 99 & 2.575 & \\
\hline
\end{tabular}

Adapted from Taylor, J.K. Quality Assurance of Chemical Measurements. Lewis Publishers, 1987.

Listed in this section are guidance for qualification for single quality-indicator samples being outside control criteria based on a $95 \%$ and $99 \%$ level of confidence. Analytical samples should not be rejected based on a singular quality control sample. Effects of other QC sample deficiencies must be considered to evaluate whether conditions are such to justify rejection of data. Appendix B provides analytical decision-making guidance for situations where multiple quality deficiencies are encountered. A strategy to incorporate into laboratory SOWs is to require corrective action if the $\pm 2.58 \sigma$ limits are exceeded. If the limits are exceeded again, data may be reported, but are subject to qualification through validation. The laboratory case narrative should reflect the corrective action.

The tests in this section are meaningful only if the radioanalytical method functions properly. If a method is deemed seriously out of control, the tests in this section are not appropriate, and no further validation needs to be done; all results may be considered unusable.

\section{Total Propagated Uncertainty (TPU)}

The tests presented in this section rely heavily upon evaluation of uncertainty associated with radioanalytical results. The factors presented in the TPU relationship in appendix D must be communicated to the laboratory prior to sample analysis in order for the laboratory to provide the relevant components of the TPU. The random factors in the TPU relationship include counting uncertainty, and net count rate uncertainty; the remaining terms comprise the systematic factors. Many laboratories choose to report uncertainties separately as total random and total systematic. These factors are acceptable to use in the tests in this section providing that the components of the uncertainties are recognized.

In the event that not all the requested uncertainties are available, the magnitude of TPU must be evaluated considering which components are the dominant factors in the relationship. At relatively low count rates, the random components will likely be the dominant factors; and at high rates, systematic components may be dominant. Communication with the laboratory in the evaluation of TPU in absence of all components is crucial in evaluating the tests presented in this section. 


\section{Standard Traceability}

Standards used in the preparation of quality control samples (laboratory control samples, matrix spikes) or sample-specific spikes (tracers or carriers) shall be shown to be traceable to a reliable source (e.g. NIST, IAEA).

\section{a. Verification}

Verification of the identity of the standard used in quality control sample preparation, or sample-specific spiking shall be performed by tracing the standard control number from the certificate to the quality control sample preparation documentation.

\section{b. Validation}

If a standard is determined to be not traceable, a qualifier is not required, but the reason code "E02" must be placed next to sample results that are in the same preparation batch with the affected QC sample.

\section{Laboratory Control Sample (LCS)}

The purpose of an LCS is to monitor the accuracy of the preparation and analysis of the analytical samples, provided that LCS is fully homogenized prior to preparation and analysis. The LCS must be the same matrix type as the analytical samples (e.g., water, soil). Exceptions should be made in cases of novel matrices (e.g. sludge, oil, biota). The spike in the LCS should be of a level near that of the analytical samples, or contractually specified at a predetermined level (e.g. 20 times the MDC may be appropriate).

Three types of LCS material have been identified as being appropriate to evaluate laboratory process accuracy:

- Traceable Reference Material (TRM)

- $\quad$ Standard Reference Material (SRM)

- Well Characterized Reference Material (WCRM)

a. Deliverables

The basic deliverables necessary to complete the checks are described in this section:

- Traceability certificate(s) for TRM and/or SRM with uncertainty associated with the standard.

- Measured result of LCS expressed in activity/unit weight or volume.

- TPU for LCS expressed in activity/unit weight or volume.

- $\mathrm{MDC}$ of LCS expressed in activity/unit weight or volume.

- Expected result of LCS expressed in activity/unit weight or volume.

- If samples within a reporting batch originate from separate preparation batches, validation requires that laboratory control sample results be included from all appropriate preparation batches. 
b. Frequency

The preparation of the LCS occurs in the laboratory simultaneously with the preparation batch of analytical samples. The LCS must be analyzed on the same detection system as the samples with which it was prepared and using the same analysis conditions as with the samples. The MDC of the LCS must be less than the specified RDL. The LCS should be prepared at a frequency of one per batch of up to 20 analytical samples.

c. Verification

Verify that one LCS was prepared at the same time with the analytical samples, is of the same matrix, and has been analyzed with an analytical batch of up to 20 analytical samples. If these criteria are not met, state as a non-correctable problem in the verification report.

d. Validation

If LCS data are not reported for a sample(s), place the reason code "L05" on the sample results for the affected preparation batch.

LCS - Test for bias

The normalized difference between the measured LCS and expected LCS results given by the following relationship is used in testing the null hypothesis that the measured and true results of the LCS do not differ significantly when compared to their respective TPU..

$$
\frac{L C S_{\text {meas. }}-L C S_{\text {exp. }}}{\sqrt{T P U_{\text {meas. }}^{2}+T P U_{\text {exp. }}^{2}}}
$$

$\mathrm{LCS}_{\text {meas. }}=$ Measured LCS result

LCS $_{\text {exp. }}=$ Expected result of LCS

$\mathrm{TPU}_{\text {meas. }}=$ Total Propagated Uncertainty of measured result

$\mathrm{TPU}_{\text {exp. }}=$ Total Propagated Uncertainty of expected result

If the normalized difference is between 1.96 and 2.58 , or between -1.96 and -2.58 , qualify sample results $\geq M D C$ "J," as the conclusion is reached that the spiked and expected results differ at the $5 \%$ level of significance. Qualify results < MDC "J" only if the normalized difference shows a negative bias. If the normalized difference is greater than 2.58 or less than -2.58 , consider the effects of deficiencies in other quality-indicator samples prior to qualifying sample results " $R$," as at the $1 \%$ level of significance, the conclusion is reached that the spiked and expected results are different enough to indicate a significant positive or negative bias. If multiple quality deficiencies are encountered, qualify using the guidance provided in Appendix B.

\section{Laboratory Duplicate}

The purpose of a laboratory duplicate is to monitor the precision of the analytical method, provided the sample is fully homogenized prior to preparation and analysis. The laboratory 
duplicate is a randomly chosen split of an analytical sample into two aliquots prior to sample preparation. To provide for relevancy of laboratory duplicate data, the sample chosen should have measurable activity (i.e. $>\mathrm{MDC}$ and $2 \sigma$ counting uncertainty); however, the test provided in this section may be performed on results $<\mathrm{MDC}$ or $2 \sigma$ counting uncertainty. In analytical methods where no sample preparation or separation is performed (e.g. gamma spectrometry), a sample recount may be performed in lieu of a laboratory duplicate, although qualification under these conditions should be based on instrumental performance, as most gamma spectrometry entails minimal sample preparation.

\section{a. Deliverables}

The basic deliverables necessary to complete the checks are described in this section:

- Measured result of sample expressed in activity/unit weight or volume.

- Measured result of duplicate expressed in activity/unit weight or volume.

- MDCs of sample and duplicate expressed in activity/unit weight or volume.

- TPU of sample and duplicate expressed in activity/unit weight or volume.

- If samples within a reporting batch originate from separate preparation batches, validation requires that laboratory duplicate results be included from all appropriate preparation batches.

b. Frequency

The preparation of the laboratory duplicate occurs in the laboratory simultaneously with the preparation batch of analytical samples. Analyses of the laboratory duplicate must proceed using the same analysis conditions used with the samples. The laboratory duplicate should be prepared at a frequency of one per preparation batch of up to 20 analytical samples.

\section{c. Verification}

Verify that one laboratory duplicate was prepared at the same time with the analytical samples, is of the same matrix, and has been analyzed with a preparation batch of up to 20 analytical samples. If there criteria are not met, state this as a non-correctable problem in the verification report.

\section{d. Validation}

If laboratory duplicate data are not reported for a sample(s), place the reason code "D04" on the sample results for the affected preparation batch.

Duplicate - Statistical test for difference between sample and duplicate

The normalized absolute difference between the sample and laboratory duplicate, given by the relationship below is used in testing the null hypothesis that the results do not differ significantly when compared to their respective TPU.

$$
\frac{S-D}{\sqrt{T P U_{S}^{2}+T P U_{D}^{2}}}
$$


$S=$ Sample result

$\mathrm{D}=$ Laboratory duplicate result

$\mathrm{TPU}_{\mathrm{s}}=$ Total Propagated Uncertainty of the sample

$\mathrm{TPU}_{\mathrm{D}}=$ Total Propagated Uncertainty of the duplicate

If the sample or laboratory duplicate results are less than their respective MDC, the results may still be used in this relationship to determine precision.

If the normalized absolute difference is greater than 1.96 , qualify the affected sample results "J," as at the $5 \%$ level of significance, the conclusion is reached that the sample and laboratory duplicate differ. If multiple quality deficiencies are encountered, qualify using the guidance provided in Appendix B.

5. Matrix Spike (MS)

The purpose of a matrix spike is to measure the effect of interferences from the sample matrix that will preclude accurate quantitation by the instrumentation, provided that samples are fully homogenized prior to preparation and analysis. The matrix spike is a split of a field-originating analytical sample in which one half of the split is spiked with a known amount of radionuclide of interest prior to sample preparation.

Due to difficulties in homogenization of solid samples for gamma analyses, a matrix spike may not present useful information. Matrix spikes may present useful information for aqueous samples; however, should not be used for energies $<100 \mathrm{KeV}$.

Matrix spikes may not be required for methods where a carrier or tracer is used, provided that the tracer chosen is chemically similar to the radionuclide of interest. Matrix effects will be detected through tracer recovery; however, difficulty may be experienced in ascertaining that poor recovery is due to matrix effect or through losses in separation.

\section{a. Deliverables}

The basic deliverables necessary to complete the checks are described in this section:

- Traceability certificate(s) for standard with uncertainty associated with the standard.

- Measured result of the unspiked sample in activity/unit weight or volume.

- Measured result of MS expressed in activity/unit weight or volume or volume

- Expected result of the MS expressed in activity/unit weight or volume.

- MDCs of unspiked sample and MS expressed in activity/unit weight or volume.

- TPU of unspiked sample, and MS expressed in activity/unit weight or volume.

- If samples within a reporting batch originate from separate preparation batches, validation requires that matrix spike results be included from all preparation batches.

\section{b. Frequency}

The preparation of the matrix spike occurs in the laboratory simultaneously with the preparation batch of analytical samples. If notably different matrices are present within the same preparation batch, matrix spikes should be prepared for each matrix. Analyses of the matrix spike must proceed using the same analysis conditions used with the samples. The 
matrix spike should be prepared at a frequency of one per preparation batch of up to 20 analytical samples.

\section{c. Verification}

Verify that one matrix spike was prepared at the same time with the analytical samples, was of the same matrix as the analytical samples, and has been analyzed with a preparation batch of up to 20 analytical samples. If this frequency is not met, state this as a non-correctable problem.

\section{d. Validation}

If matrix spike data are not reported for a sample(s), place the reason code "M05" on the sample results for the affected preparation batch.

Matrix Spike - Test for matrix-induced bias

The normalized difference between the spike result (SSR-SR) and expected value (ER) of the MS given by the following equation is used in testing the null hypothesis that the expected and measured results of the MS do not differ significantly when compared to their respective TPU.

$$
\frac{(S S R-S R)-E R}{\sqrt{T P U_{S S R}^{2}+T P U_{S R}^{2}+T P U_{E R}^{2}}}
$$

$$
\begin{array}{ll}
\text { SSR } & =\text { Spiked Sample Result } \\
\text { SR } & =\text { Sample Result (unspiked) } \\
\text { ER } & =\text { Expected Result (spike amount) } \\
\text { TPU } & =\text { Total Propagated Uncertainty }
\end{array}
$$

If the normalized difference is between 1.96 and 2.58 , or between -1.96 and -2.58 , qualify sample results $\geq$ MDC "J," as the conclusion is reached that the spiked and expected results differ at the 5\% level of significance. Qualify sample results < MDC "UJ" only if the normalized difference shows a negative bias. If the normalized difference is greater than 2.58 or less than -2.58 , consider the effects of deficiencies in other quality-indicator samples prior to qualifying sample results " $R$," as at the level of significance of $1 \%$, the conclusion is reached that the spiked and expected results differ to a point indicative of a significant positive or negative matrix-induced bias.

Consideration should be given to the similarity in matrix type among samples in the preparation batch batches. If the matrices differ notably (particularly in soil particle size) qualification may be placed on only the sample associated with the matrix spike. If matrices do not differ notably, qualification may be placed on all samples in the preparation batch. If multiple quality deficiencies are encountered, qualify using the guidance provided in Appendix B. 


\section{Method Blank}

The purpose of a method blank is to monitor the presence of contamination of the analyte of interest in the sample preparation and analysis processes. The method blank is a laboratory-generated sample of the same matrix as the analytical samples but in absence of the analyte of interest.

\section{a. Deliverables}

The basic deliverables necessary to complete the checks are described in this section:

- Measured result of method blank expressed in activity/unit weight or volume.

- $\quad$ MDC of method blank expressed in activity/unit weight or volume.

- TPU of method blank expressed in activity/unit weight or volume.

- If samples within a reporting batch originate from separate preparation batches, validation requires that method blank results be included from all preparation batches.

\section{b. Frequency}

The preparation of the method blank occurs in the laboratory simultaneously with the preparation batch of analytical samples. Analyses of the method blank must proceed using the same analysis conditions used with the samples. The method blank should be prepared at a frequency of one per batch of up to 20 analytical samples.

\section{c. Verification}

Verify that all method blank activities are less than their MDC and $2 s$ counting uncertainty. If method blank activity is greater than the MDC or $2 s$ counting uncertainty, note this in the verification report.

Verify that one method blank was prepared at the same time with the analytical samples, is of the same matrix, and has been analyzed at a frequency of one per preparation batch of up to 20 analytical samples. If these criteria are not met, state this as a non-correctable problem in the verification report.

\section{d. Validation}

If method blank data are not reported for a sample(s), place the reason code "B06" on the sample results for the affected preparation batch. .

If either of the following conditions are met, no actions outlined in this section need to be performed:

- method blank result is less than its MDC or less than its 2 s counting uncertainty

- method blank result is greater than its MDC with the sample result less than its MDC.

Validation of the method blank tests whether blank contamination is of a level which may be indicative of systematic contamination through sample preparation. 
If both the method blank and sample activity are greater than their respective MDC or $2 \mathrm{~s}$ counting uncertainty, the following test shall be used in determining the effect of possible blank contamination on sample results.

The normalized absolute difference between the method blank and a sample result, given by the relationship below, is used in testing the null hypothesis that the sample and the method blank do not differ significantly when compared to their respective TPU.. This test may be used as long as the method blank is reported in terms of activity per unit weight or volume consistent with the sample results.

$$
\frac{|S-B|}{\sqrt{T P U_{S}^{2}+T P U_{B}^{2}}}
$$

$\mathrm{S}=$ Sample result

$\mathrm{B}=$ Method blank result

TPU $=$ Total Propagated Uncertainty

If the normalized absolute difference is greater than 2.58 , no qualification is necessary, as at the $1 \%$ level of significance, the conclusion is reached that the method blank and sample differ significantly. If the normalized absolute difference is between 1.96 and 2.58, qualify sample results $\geq \mathrm{MDC}$ "J," the sample and method blank differ at the $5 \%$ level of significance (sample results $<\mathrm{MDC}$ do not require qualification). If the normalized absolute difference is between 0 and 1.96, consider the effects of deficiencies in other quality-indicator samples prior to qualifying sample results " $R$," the conclusion is reached that the method blank and sample results differ at the $1 \%$ level of significance. If multiple quality deficiencies are encountered, qualify using the guidance provided in Appendix B.

F. Chemical Yield - Tracers and Carriers

Tracers and carriers are used in radiochemical separations methods to provide evaluation of chemical separation. Chemical yield is evaluated through the recovery of chemical species spiked into samples. Yield is evaluated radiometrically with a tracer and gravimetrically with a carrier. Each sample is spiked with either a carrier or tracer, and sample results are adjusted for yields greater or less than $100 \%$.

Generally, a low yield is indicative of losses of tracer and radionuclide of interest through sample separation, and recoveries greater than expected $(>100 \%)$ are indicative of instrumental problems or contamination, as carriers fortified into samples are not expected to be recovered at levels greater than spiked.

\section{Verification}

Verify that for applicable analyses, one carrier or tracer recovery is reported for each sample. If a carrier or tracer percent recovery is not reported for each sample, contact the laboratory for submittal of this data. If the data can not be provided, state this as a non-correctable problem in the verification report. 
As yield decreases, the MDC may elevate to a point at which the RDL is exceeded, and analytical results are contractually noncompliant. If the laboratory has not initiated corrective action, for samples in which the MDC exceeds the RDL, the project may choose to contact the laboratory for sample rework. If rework is not feasible, indicate the noncompliant data in the verification report.

2. Validation

If yield is not reported for a sample(s), place the reason code "Y04" on the sample result.

Yield is validated based on percent recovery of the spiked nuclide. Low yield may be indicative of increased uncertainty in the sample result. Criteria for qualification should be based on what magnitude of correction has been applied to the sample result (e.g. $20 \%$ recovery implies a sample result correction of 5), although a point of debate exists concerning useability of radionuclide data with yields near $0 \%$. Yield criteria may also be established from existing sample yield data from previous sampling at the site, if this data are available.

Sample results should not be qualified based on yield alone. Sample yield should be evaluated in reference to chemical yield of quality-indicator samples. If yield is generally low throughout the preparation batch, but recoveries of target radionuclides in the LCS are acceptable, data may be accepted without qualification; however, if quality control sample yield is generally low, sample results with low yield may need qualification.

G. Required Detection Limits (RDL)

\section{Verification}

For all samples, the MDC must be less than the contractual RDL. RDLs are identified and communicated to the laboratory in the laboratory SOW. If the MDC is greater than the RDL, this may indicate use of a small sample size, inadequate count times, or matrix problems. All sample results shall be reviewed to determine RDL compliance. In cases where the MDC is greater than the RDL, the verifier should consult the laboratory case narrative to evaluate the reason for the noncompliance; the project may request a reanalysis.

H. Nuclide Identification and Quantification

1. Test for Detection

a. Validation

The test for detection of a radionuclide includes two distinct steps.

i. The first step is to evaluate whether a sample result is greater than its MDC.

ii. The second step is to evaluate whether the sample result is greater than its $2 \mathrm{~s}$ counting uncertainty.

If the sample result is not greater than its MDC or less than its $2 s$ counting uncertainty, qualify the sample result "U." 
If the sample result is less than its MDC (assuming that the MDC is reported at the $95 \%$ confidence level) the data user is accepting the probability of $5 \%$ of a false negative result. If the sample result is less than its $2 \mathrm{~s}$ counting uncertainty, at the $95 \%$ confidence level, the radionuclide result is not different from zero.

In placing the "U" qualifier, special attention must be paid to instances where nominal conditions are not followed (i.e. sample aliquot size, count time). Sample results which are less than their MDC, but greater than their $2 \mathrm{~s}$ counting uncertainty may have been counted for a period of time long enough to be considered detected. Sample results which are greater than the MDC, but less than the $2 \mathrm{~s}$ counting uncertainty may not have been counted for a period of time long enough to be considered detected.

2. Negative Results

a. Validation

Negative results that have absolute values greater than their $2 \mathrm{~s}$ counting uncertainty are an indication that the instrument background has shifted. The implication of unstable background is a possible negative bias in the sample result. Qualification due to very negative sample results must take into account evaluation of calibration verification background and quality-indicator samples that may indicate a bias. Qualifiers should be placed using professional judgement when applied due to the occurrence of negative results, and the rationale for placement must be fully described in the validation report.

\section{Sample Result Recalculation}

The accuracy and consistency of sample result calculation by the laboratory can be addressed through two different techniques. The application of each strategy depends on the laboratory's ability to minimize transcription during reporting, and how familiar the project is with the performance of the laboratory.

a. If sample results are produced primarily through software processing and minimal transcription is performed in the laboratory, the data system(s) can be evaluated during an audit or surveillance by performing two different tests on the software: 1) supply the data system a consistent set of input designed to provide a consistent set of output, and 2) supply the data system a set of nonconforming data to test the error detection routines. An evaluation of the laboratory's software configuration control and security is also necessary. If reporting software has been developed for customized use in the laboratory, additional confidence is gained through the laboratory's adherence to the IEEE Standard for Software Verification and Validation Plans 1012-1986 and through maintenance of a software verification and validation plan. Through this technique, a high level of confidence can be gained in the laboratory's reporting techniques and will result in a minimal need for manual recalculation of sample results.

b. If the laboratory has a high rate of manual transcription in generation of sample results, the project may choose to manually recalculate sample results at a determined frequency. If sample results cannot be reproduced through manual calculation, contacting the laboratory may be necessary to resolve the problem. Qualifiers should be placed using professional judgement. 
Activity and TPU equations provided in appendix $\mathrm{C}$ are useful for providing the basic structure for calculating radioanalytical results. Modifications to the equations may be needed in method-specific cases. All calculations used must be contractually agreed upon between the project and the laboratory prior to sample analysis, and iterated in the laboratory SOW.

I. Instrument-Specific Sample Considerations

1. Sample Analytical Parameters for Gas Proportional Counting

a. Validation

Equations to approximate a parametric relationship between standard weight and activity are detector-specific. They are developed from the mass attenuation curves by the laboratory and are applied to calculate sample-specific efficiencies. If recalculating sample results, efficiencies used must be sample-specific.

A representative sample aliquot must be chosen to ensure the dissolved solid content of the sample falls within the mass range of the appropriate curve. Sample results for which aliquot weight is outside the attenuation curve should be qualified " $\mathrm{J}$ " if not reanalyzed with a smaller aliquot.

2. Spectral Interpretation (alpha and gamma spectrometry)

\section{a. Gamma Spectrometry}

Identification of the radionuclides associated with the measured gamma-ray energies and intensities is largely performed by gamma-ray spectral-analysis algorithms. These algorithms automatically locate peaks in spectra by applying a user-definable set of criteria. Based upon this set of criteria, the peaks in a spectrum are distinguished from the Compton edges, backscatter peaks, and the numerous features created by the background radiation and detection process. The algorithms automatically calculate the peak areas and correct them for the system's detection efficiency as a function of gamma energy. The identification of the radionuclides that produced the peaks is accomplished by comparison to the information in a customizable radionuclide analysis library. For all peaks identified in a spectrum (that correspond to a radionuclide in the analysis library), the algorithms calculate the activities of the identified radionuclides in the sample. However, the use of peak search, identification, and quantification algorithms does not preclude the need for laboratory data review by an experienced spectroscopist.

The resulting quality of the gamma spectrometry data will largely depend upon the ability of the spectroscopist to establish a set of analysis criteria to be used by the algorithms with perform the location, identification and quantification of features within spectra. The quality of this process is greatly influenced by the spectral library. For this reason, it is recommended that the library originate from a reliable source and software be controlled with respect to changes in identification criteria. Multiple libraries are often constructed based upon expected interferences and the level of activity in the samples. The multiple libraries reduce the number of unidentified peaks due to summation events and interferences within the sample matrix. The goal of reducing the number of unidentified peaks within a spectrum, while not compromising the analysis routines by the inclusion of extremely low abundanice 
branching probabilities, helps limit the uncertainty of detection of those radionuclides which emit more than one photon.

The data package should include both identified and unidentified peaks. This will support the evaluation of instrumental system control.

\section{b. Evaluation of Gamma Spectra}

The assurance of radionuclide identification and quantitation by properly functioning algorithms, through reasonable resolution criteria, and by an evaluation by an experienced radiochemist will provide a level of certainty in the quality of the data. Validation of data produced under these conditions should be minimal, i.e. a thorough review of all spectra including radionuclide identification and recalculation of sample results should not be necessary. A recommendation is to browse sample-specific spectra for changes in energies positions of target radionuclides and for significant peak overlap.

Radiochemical data produced under substandard or unknown conditions for identification and quantification warrant a closer inspection. Recommended criteria for evaluating gamma spectra in relation to radionuclide identification and resolution are as follows:

i. Where more than one isotope of a single Naturally Occurring Radioactive Material (NORM) series are reported, reported results for that series should demonstrate secular equilibrium.

ii. For soil samples, two peaks that are almost always observed are the $511 \mathrm{keV}$ annihilation peak and the $1460 \mathrm{keV}$ peak of ${ }^{40} \mathrm{~K}$. The appearance of these peaks at the respective energy, and the respective peak shape should be checked.

iii. If ${ }^{40} \mathrm{~K}$ is quantitated in the analysis, the reported value should be checked against the activity expected in site's soil (if those data are available).

iv. For isotopes identified and/or quantitated with two or more gamma energies (i.e. 1173 and $1332 \mathrm{keV}$ for ${ }^{60} \mathrm{Co}$ ) the count rate at each energy can be observed to ensure that the count rate at each respective energy is reasonable to confirm the presence of the isotope.

\section{c. Alpha Spectra}

As with gamma spectrometry, the alpha spectrometry can require the use of algorithms for the location, identification, and quantification of peaks created by radionuclides with spectra. While many of the analysis algorithms are the same for alpha spectrometry as gamma spectrometry, there are fundamental differences which effect the validation of data. These differences which are inherent to the mode of the decay, sample preparation techniques, and phenomena associated to the detection and quantification require validation. As with gamma spectrometry, if algorithms and operational criteria are employed by the laboratory, then they should be reviewed by a competent spectroscopist and/or radiochemist.

Alpha spectrometry has limitations due to the alpha particles ability to transfer energy at an extremely high rate. This phenomenon is responsible for alpha particles being significantly attenuated by the sample matter, the mass of the radionuclide itself, and the molecules of air 
between the detector and the sample. If the mass of the samples is not reduced or the radionuclides are present in appreciable amounts, then likelihood of receiving meaningful data is reduced due to attenuation. Therefore, samples for alpha spectrometry frequently require extensive radiochemical preparations, with the sample being placed in vacuums and in close proximity to the detectors. Typically, these separation/preparation techniques isolate one or two target nuclides, thus reducing the possible attenuation which occurs at high masses.

This separation process presents the possibility that contaminating elements could break through the chemical separation process. If chemical breakthrough were to occur, these interferences could erroneously be incorporated into the quantification of the radioisotope of interest.

Unlike gamma spectrometry, the spectra resulting from properly prepared alpha sources/samples are relatively simple, since the peaks are near Gaussian, the background is very low, and detector efficiency is almost uniform for the $3.0 \mathrm{MeV}$ to $6.5 \mathrm{MeV}$ energy range. Frequently, the use of Range of Interest (ROI) are integrated to determine gross activity which can be adjusted for background and then corrected for chemical recovery, decay, aliquot, and detection efficiency to produce concentration. A ROI peak report, including all information needed to manually recalculate results, is useful to evaluate data, as well as the spectra itself.

\section{d. Evaluation of Alpha Spectra}

Alpha spectra is not generated through the application of algorithms; consequently the approach to validation differs from that of gamma spectrometry. The following two tests should be performed on alpha spectral data:

i. target peaks should be in the energy range of interest

ii. peak tailing should not significantly overlap peaks at lower energies.

Professional judgement should be used in qualification of sample data based on spectral interferences or peak misidentification. Communication with the laboratory is essential to obtaining reliable identification/quantitation of suspect radionuclides.

\section{APPENDIXES}

Appendix A Data Validation Qualifiers and Reason Codes

Appendix B Mapping for Multiple Quality Deficiencies

Appendix C Calculations and Equations

Appendix D Listing of Common Nuclides/Methodologies

Appendix E Recommended Surveillance Considerations for Gamma Algorithms 
APPENDIX A

DATA VALIDATION QUALIFIERS AND REASON CODES 


\title{
$\underline{\text { Radiological Analytical Data }}$
}

Reason codes must be used with all qualifiers placed on analytical data.

U Nuclide considered not detected above the reported MDC or $2 \sigma$ counting uncertainty

J Nuclide identified; the associated numerical value is approximated

UJ Nuclide not detected above the reported MDC or $2 \sigma$ counting uncertainty and a quality deficiency affects the data making the reported data more uncertain

$\mathrm{R} \quad$ Result is not usable for its intended purpose

$=\quad$ "Equals" sign, indicates that no qualifier is necessary

\section{DATA VALIDATION QUALIFICATION CODES}

\author{
$\underline{\text { Radiological Analytical Data }}$
}

\section{Method Blank}

B01 Concentration of contaminant in the method blank at a level $\Rightarrow$ the qualification level.

B02 Method blank was not the same matrix as the analytical samples.

B03 Gross contamination exists.

B04 Blanks were not analyzed at appropriate frequency.

B05 Sample not significantly different than radiochemical method blank.

B06 Blank data not reported.

B07 Other (describe in comments)

Calibration

$\mathrm{C} 01$ Initial calibration sequence was not followed as appropriate.

$\mathrm{C} 02$ Calibration was not performed at the appropriate frequency.

C03 Calibration data not reported

C04 Calibration not performed.

C05 Chemical resolution criteria were not satisfied.

C06 Standard curve was established with fewer than the required number of standards

$\mathrm{C} 07$ Instrumental system determined to be out of control

C08 Other (describe in comments)

\section{Laboratory Duplicate}

D01 Significant difference between sample and duplicate.

D02 Laboratory duplicate was not analyzed at the appropriate frequency

D03 Laboratory duplicate data not reported

D04 Other (describe in comments). 
Evidentiary Concerns

E01 Custody of Sample in Question.

E02 Standard not Traceable.

E03 Other (describe in comments)

General

G01 Professional judgement was used to qualify the data.

G02 Other (describe in comments)

Holding Times

H01 Holding times were exceeded.

$\mathrm{H} 02$ Holding times were grossly exceeded.

H03 Samples were not preserved properly.

H04 Other (describe in comments)

Laboratory Control Sample

L01 LCS recovery above upper control limit.

L02 LCS recovery below lower control limit.

L03 LCS was not analyzed at appropriate frequency.

L04 LCS not the same matrix as the analytical samples.

L05 LCS data not reported

L06 Other (describe in comments)

Matrix Spike and MS/MSD

M01 MS recovery above upper control limit.

M02 MS recovery below lower control limit.

M03 MS not analyzed at the appropriate frequency

M04 MS data not reported

M05 Other (describe in comments)

Instrument Performance

P01 High background levels or a shift in the energy calibration were observed

P02 Extraneous peaks were observed.

P03 Loss of resolution was observed.

P04 Peak-tailing or peak splitting that may result in inaccurate quantitation were observed

P05 Instrument performance not analyzed at the appropriate frequency

P06 Other (describe in comments)

Quantitation

Q01 Peak Misidentified

Q02 Target analyte affected by interfering peak.

Q03 Qualitative criteria were not satisfied.

Q04 Cross contamination occurred.

Q05 No raw data were provided to confirm quantitation. 
Q06 MDC.> RDL

Q07 Inappropriate aliquot sizes were used.

Q08 Sample result $<$ MDC.

Q09 Sample result $<2$ s uncertainty.

Q10 Negative result.

Q11 Compounds were not adequately resolved.

Q12 Sample geometry different from calibration geometry.

Q13 Sample weight greater than greatest weight on mass attenuation curve.

Q14 Other (describe in comments)

Radiochemical Yield

Y01 Radiochemical tracer yield was above the upper control limit

Y02 Radiochemical tracer yield was below the lower control limit

Y03 Radiochemical tracer yield was zero.

Y04 Radiochemical yield data was not present.

Y05 Other (describe in comments) 


\section{APPENDIX B}

MAPPING FOR MULTIPLE QUALITY DEFICIENCIES 

These tables represent validation qualification decisions made based on radioanalytical data quality considerations only. Data quality needs (e.g. risk assessment, remediation technologies) must be considered when using the guidance in these tables. For example, quantitative data needs may necessitate that data be rejected due to multiple quality deficiencies, but qualititative data needs may indicate that the same data should only be qualified estimated.

Table table B.1 provides a mapping scenario for qualification guidance. For example, if the Laboratory Control Sample shows a high bias, and chemical yield is also high, choose the letters " $A$ " and "E" and reference Table B.2 for guidance on qualification based on those quality deficiencies. The differing separation lines in table B.1 indicate relationships among the quality indicators QC samples and yield. Double lines indicate an "and" function, in that any combination is possible. Thick lines indicate that a single QC sample can only indicate a bias in one direction.

Table B.1

\begin{tabular}{|c|c|c|c|c|c|c|c|}
\hline \multicolumn{2}{|c|}{$\begin{array}{l}\text { Lab Control } \\
\text { Sample }\end{array}$} & \multicolumn{2}{|c|}{ Matrix Spike } & \multicolumn{2}{|c|}{ Chemical Yield } & \multirow[t]{2}{*}{$\begin{array}{l}\text { Method } \\
\text { Blank }\end{array}$} & \multirow[t]{2}{*}{$\begin{array}{c}\text { Lab } \\
\text { Duplicate }\end{array}$} \\
\hline $\begin{array}{l}\text { High } \\
\text { Bias }\end{array}$ & $\begin{array}{l}\text { Low } \\
\text { Bias }\end{array}$ & High Bias & Low Bias & High & Low & & \\
\hline $\mathrm{A}$ & B & C & $\mathrm{D}$ & $E$ & $\mathrm{~F}$ & $G$ & $\mathrm{H}$ \\
\hline
\end{tabular}


B-4

Table B.2. Qualification Guidance for 2 Simultaneous Quality Deficiencies

\begin{tabular}{|c|c|c|}
\hline \multirow[t]{2}{*}{ Combination } & \multicolumn{2}{|c|}{ Qualification } \\
\hline & $<\mathrm{MDC}$ & $\geq \mathrm{MDC}$ \\
\hline $\mathrm{AC}$ & none & $\mathrm{R}$ \\
\hline $\mathrm{AD}$ & UJ & $\mathbf{J}$ \\
\hline $\mathrm{AE}$ & none & $\mathrm{R}$ \\
\hline $\mathrm{AF}$ & UJ & $\mathbf{J}$ \\
\hline $\mathrm{AG}$ & none & $\mathrm{J}$ \\
\hline $\mathrm{AH}$ & none & $\mathrm{J}$ \\
\hline $\mathrm{BC}$ & UJ & $\mathrm{J}$ \\
\hline $\mathrm{BD}$ & $\mathbf{R}$ & $\mathrm{J}$ \\
\hline $\mathrm{BE}$ & UJ & $\mathrm{J}$ \\
\hline $\mathrm{BF}$ & $\mathbf{R}$ & $\mathrm{J}$ \\
\hline BG & UJ & $\mathbf{J}$ \\
\hline $\mathrm{BH}$ & UJ & $\mathrm{J}$ \\
\hline $\mathrm{CE}$ & none & $\mathrm{R}$ \\
\hline $\mathrm{CF}$ & UJ & $\mathrm{J}$ \\
\hline CG & none & $\mathrm{R}$ \\
\hline $\mathrm{CH}$ & none & $\mathrm{R}$ \\
\hline $\mathrm{DE}$ & UJ & $\mathbf{J}$ \\
\hline DF & $\mathrm{R}$ & $\mathbf{J}$ \\
\hline DG & UJ & $\mathrm{R}$ \\
\hline $\mathrm{DH}$ & UJ & $\mathbf{R}$ \\
\hline EG & none & $\mathbf{R}$ \\
\hline $\mathrm{EH}$ & none & $\mathrm{J}$ \\
\hline FG & UJ & $\mathrm{J}$ \\
\hline $\mathrm{FH}$ & UJ & $\mathrm{J}$ \\
\hline $\mathrm{GH}$ & UJ & $\mathrm{J}$ \\
\hline
\end{tabular}


Table B.3. Qualification Guidance for 3 Simultaneous Quality Deficiencies

\begin{tabular}{|c|c|c|}
\hline \multirow[t]{2}{*}{ Combination } & \multicolumn{2}{|c|}{ Qualification } \\
\hline & $<\mathrm{MDC}$ & $\geq \mathrm{MDC}$ \\
\hline $\mathrm{ACE}$ & none & $\mathrm{R}$ \\
\hline $\mathrm{ACF}$ & $\mathrm{UJ}$ & $\mathbf{R}$ \\
\hline ACG & none & $\mathrm{R}$ \\
\hline $\mathrm{ACH}$ & none & $\mathrm{R}$ \\
\hline $\mathrm{ADE}$ & UJ & $\mathrm{R}$ \\
\hline $\mathrm{ADF}$ & $\mathrm{R}$ & $\mathrm{R}$ \\
\hline $\mathrm{ADG}$ & UJ & $\mathrm{R}$ \\
\hline $\mathrm{ADH}$ & UJ & $\mathrm{R}$ \\
\hline AEG & none & $\mathrm{R}$ \\
\hline $\mathrm{AEH}$ & none & $\mathrm{R}$ \\
\hline AFG & UJ & $\mathrm{R}$ \\
\hline $\mathrm{AFH}$ & none & $\mathrm{R}$ \\
\hline $\mathrm{BCE}$ & UJ & $\mathrm{R}$ \\
\hline $\mathrm{BCF}$ & UJ & $\mathrm{R}$ \\
\hline $\mathrm{BCG}$ & UJ & $\mathrm{R}$ \\
\hline $\mathrm{BCH}$ & UJ & $\mathrm{R}$ \\
\hline $\mathrm{BDE}$ & $\mathbf{R}$ & $\mathrm{R}$ \\
\hline BDF & $\mathrm{R}$ & $\mathrm{R}$ \\
\hline $\mathrm{BDG}$ & $\mathbf{R}$ & $\mathrm{R}$ \\
\hline $\mathrm{BDH}$ & $\mathrm{R}$ & $\mathrm{R}$ \\
\hline BEG & UJ & $\mathrm{R}$ \\
\hline BEH & UJ & $\mathrm{R}$ \\
\hline $\mathrm{BFG}$ & $\mathrm{R}$ & $\mathbf{R}$ \\
\hline $\mathrm{BFH}$ & $\mathrm{R}$ & $\mathrm{R}$ \\
\hline CEG & none & $\mathrm{R}$ \\
\hline $\mathrm{CEH}$ & none & $\mathrm{R}$ \\
\hline $\mathrm{CFG}$ & UJ & $\mathrm{R}$ \\
\hline $\mathrm{CFH}$ & UJ & $\mathbf{R}$ \\
\hline DEG & UJ & $\mathrm{R}$ \\
\hline DEH & UJ & $\mathrm{R}$ \\
\hline DFG & $\mathrm{R}$ & $\mathbf{R}$ \\
\hline DFH & $\mathrm{R}$ & $\mathrm{R}$ \\
\hline EGH & none & $\mathrm{R}$ \\
\hline FGH & UJ & $\mathrm{R}$ \\
\hline
\end{tabular}


B-6

Table B.4. Qualification Guidance for 4 Simultaneous Quality Deficiencies

\begin{tabular}{|c|c|c|}
\hline \multirow[t]{2}{*}{ Combination } & \multicolumn{2}{|c|}{ Qualification } \\
\hline & $<\mathrm{MDC}$ & $\geq \mathrm{MDC}$ \\
\hline ACEG & none & $\mathbf{R}$ \\
\hline ACFG & UJ & $\mathrm{R}$ \\
\hline $\mathrm{ACEH}$ & none & $\mathrm{R}$ \\
\hline $\mathrm{ACFH}$ & UJ & $\mathrm{R}$ \\
\hline$A D E G$ & UJ & $\mathrm{R}$ \\
\hline ADFG & $\mathrm{R}$ & $\mathbf{R}$ \\
\hline ADEH & UJ & $\mathrm{R}$ \\
\hline ADFH & $\mathrm{R}$ & $\mathrm{R}$ \\
\hline BCEG & UJ & $\mathrm{R}$ \\
\hline BCFG & $\mathrm{R}$ & $\mathbf{R}$ \\
\hline BCEH & UJ & $\mathrm{R}$ \\
\hline $\mathrm{BCFH}$ & $\mathrm{R}$ & $\mathrm{R}$ \\
\hline BDEG & $\mathrm{R}$ & $\mathbf{R}$ \\
\hline BDFG & $\mathrm{R}$ & $\mathbf{R}$ \\
\hline $\mathrm{BDEH}$ & $\mathrm{R}$ & $\mathbf{R}$ \\
\hline BDFH & $\mathrm{R}$ & $\mathbf{R}$ \\
\hline CEGH & none & $\mathrm{R}$ \\
\hline $\mathrm{CFGH}$ & UJ & $\mathbf{R}$ \\
\hline DEGH & UJ & $\mathrm{R}$ \\
\hline DFGH & $\mathrm{R}$ & $\mathrm{R}$ \\
\hline
\end{tabular}




\section{APPENDIX C}

CALCULATIONS AND EQUATIONS 


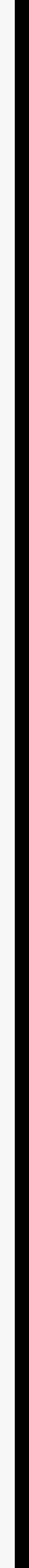


The equations in this appendix are meant to be used as the basis for developing methodspecific applications. The appendix is divided in the following manner:

- Pages 3-8 Provide Equations For Method Blank Corrected Sample Concentrations

- Pages 9-14 Provide Equations For Sample Concentrations Without Blank Subtraction

Sample Activity Concentration - (Method Blank Corrected Sample Concentrations)

$$
A C T_{s}=\frac{N C R_{s}}{2.22 * E F F * A L I * R * A B N_{s} * \mathrm{e}^{-\lambda . t} * C F}
$$

Where: $\quad \mathrm{ACT}_{\mathrm{s}}=$ Sample Activity Concentration $(\mathrm{pCi} / \mathrm{g}$ or $\mathrm{pCi} / \mathrm{L})$

$\mathrm{NCR}_{\mathrm{S}}=$ Net Sample Count Rate in $\mathrm{cpm}$

$2.22=$ Factor for Converting $\mathrm{dpm}$ to $\mathrm{pCi}$

$\mathrm{EFF}=$ Detector Efficiency (Fraction)

$\mathrm{ALI}=$ Sample Aliquot Volume or Mass ( $\mathrm{g}$ or $\mathrm{L}$ )

$\mathrm{ABN}_{\mathrm{S}}=$ Abundance Fraction of the Emissions Used for Analyte Identification/Quantification

$\mathrm{R}=$ Sample Tracer/Carrier (Chemical) Recovery

$\lambda=$ Analyte Decay Constant $-\ln 2 /$ (half-life) [Same units as the half-life used to compute $\lambda]$

$\mathrm{t}=$ Time from Sample Collection to Radionuclide Separation or Mid-point of Count Time (Same units as half-life)

$\mathrm{CF}=$ Other Correction Factors as Appropriate (i.e., Ingrowth factor, Self-absorption Factor, etc.) 
Net Sample Count Rate (NCR) and $\sigma_{\mathrm{NCR}}$ - (Method Blank Corrected Sample Concentrations)

$$
\begin{aligned}
& N C R=\left(\frac{C_{G S}}{T_{G S}}-\frac{C_{S B}}{T_{S B}}\right)-\left\lfloor\left(\frac{C_{G B}}{T_{G B}}-\frac{C_{B B}}{T_{B B}}\right) *\left(\frac{E F F_{S D}}{E E F_{B D}}\right) *\left(\frac{R_{S}}{R_{B}}\right)\right\rfloor \\
& \text { Where: } \\
& \mathrm{C}_{\mathrm{GS}}=\text { Sample Counts } \\
& \mathrm{T}_{\mathrm{GS}}=\text { Sample CountTime (minutes) } \\
& \mathrm{C}_{\mathrm{SB}}=\text { Background Counts } \\
& \mathrm{T}_{\mathrm{SB}}=\text { Background CountTime (minutes) } \\
& \mathrm{C}_{\mathrm{GB}}=\text { Gross Method Blank Counts } \\
& \mathrm{T}_{\mathrm{GB}}=\text { Gross Method Blank CountTime (minutes) } \\
& \mathrm{C}_{\mathrm{BB}}=\text { Method Blank Background Counts } \\
& \mathrm{T}_{\mathrm{BB}}=\text { Method Blank Background CountTime (minutes) } \\
& \mathrm{EFF}_{\mathrm{SD}}=\text { Efficiency of the Sample Detector } \\
& \mathrm{EFF}_{\mathrm{BD}}=\text { Efficiency of the Method Blank Detector } \\
& \mathrm{R}_{\mathrm{S}}=\text { Sample Tracer/Carrier Recovery Fraction } \\
& \mathrm{R}_{\mathrm{B}}=\text { Method Blank Tracer/Carrier Recovery Fraction } \\
& \sigma_{N C R}^{2}=\left[\frac{C_{G S}}{T_{G S}^{2}}+\frac{C_{S B}}{T_{S B}^{2}}\right]+\left\{\left[\frac{C_{G B}}{T_{G B}^{2}}+\frac{C_{B B}}{T_{B B}^{2}}\right] *\left(\frac{E F F_{S D}}{E F F_{B D}}\right)^{2} *\left(\frac{R_{S}}{R_{B}}\right)^{2}\right\} \\
& +\left\{\left[\left(\frac{C_{G B}}{T_{G B}}-\frac{C_{B B}}{T_{B B}}\right) *\left(\frac{E E F_{S D}}{E F F_{B D}}\right) *\left(\frac{R_{S}}{R_{B}}\right)\right]^{2} *\left[\left(\frac{\sigma_{E F F_{S D}}}{E E F_{S D}}\right)^{2}+\left(\frac{\sigma_{E F F_{S D}}}{E E F_{B D}}\right)^{2}+\left(\frac{\sigma_{R_{S}}}{R_{S}}\right)^{2}+\left(\frac{\sigma_{R_{B}}}{R_{B}}\right)^{2}\right]\right\} \\
& \sigma_{N C R}=\sqrt{\sigma_{N C R}^{2}}
\end{aligned}
$$




$$
R=\frac{\left(\frac{C_{G T}}{T_{G T}}-\frac{C_{T B}}{T_{T B}}\right)}{E E F * A B N_{T} * A M T_{T}}=\frac{N C R_{T}}{E F F * A B N_{T} * A M T_{T}}
$$

Where:

$$
\begin{aligned}
\mathrm{R} & =\text { Tracer Recovery } \\
\mathrm{C}_{\mathrm{GT}} & =\text { Gross Count of Tracer } \\
\mathrm{T}_{\mathrm{GT}} & =\text { Tracer CountTime (minutes) } \\
\mathrm{C}_{\mathrm{TB}} & =\text { Background Count of Tracer [Region of Interest (ROI) }] \\
\mathrm{T}_{\mathrm{TB}} & =\text { Background CountTime (minutes) } \\
\mathrm{EFF} & =\text { Detector Efficiency Fraction } \\
\mathrm{AMT}_{\mathrm{T}}= & \text { Amount of Tracer Activity Added (dpm) } \\
\mathrm{NCR}_{\mathrm{T}}= & \text { Net Count Rate of Tracer (cpm) } \\
\mathrm{ABN}_{\mathrm{T}}= & \text { Abundance Fraction of the Tracer Emissions used for } \\
& \text { Quantification of the Tracer }
\end{aligned}
$$

Note: It is assumed that the tracer half-life is long enough to be an insignificant uncertainty contributor. If the tracer has a relatively short half-life, then it must be considered and these equations modified. Likewise, uncertainty in the time is also considered to be an insignificant contributor. 
Calculation of $\mathbf{R E}_{\mathrm{R}}$ - Radiometric - (Method Blank Corrected Sample Concentrations)

$$
\begin{gathered}
\left(R E_{R}\right)^{2}=\left(\frac{\sigma_{R}}{R}\right)^{2}=\left(\frac{\sigma_{N C R_{T}}}{N C R_{T}}\right)^{2}+\left(\frac{\sigma_{E F F}}{E F F}\right)^{2}+\left(\frac{\sigma_{A M T_{T}}}{A M T_{T}}\right)^{2} \\
\left.\left(\frac{\sigma_{N C R_{T}}}{N C R_{T}}\right)^{2}=\frac{\left(\frac{C_{G T}}{T_{G T}^{2}}+\frac{C_{T B}}{T_{T B}^{2}}\right)^{N C R_{T}^{2}}}{\sigma_{A M T_{T}}}\right)^{2}=\left(\frac{\sigma_{S T S_{T}}}{S T S_{T}}\right)^{2}+\left(\frac{\sigma_{M A S S_{T}}}{M A S S_{T}}\right)^{2}+\left(\frac{\sigma_{V O L_{T}}}{V O L_{T}}\right)^{2}+\Sigma\left(\frac{\sigma_{D I L_{T}}}{D I L_{T}}\right)^{2}+\Sigma\left(\frac{\sigma_{A L I_{T}}}{A L I_{T}}\right) \\
R E_{R}=\sqrt{\left(\frac{\sigma_{R}}{R}\right)^{2}}
\end{gathered}
$$

Where:

$$
\begin{aligned}
\mathrm{RE}_{\mathrm{R}}= & \text { Relative Error of the Tracer Recovery } \\
\sigma_{\mathrm{R}}= & \text { Standard Deviation of the Tracer Recovery } \\
\sigma_{\mathrm{NCR}}= & \text { Standard Deviation of the Tracer's Net Count Rate } \\
\sigma_{\mathrm{EFF}}= & \text { Standard Deviation of the Detector Efficiency } \\
\sigma_{\mathrm{AMT}}= & \text { Standard Deviation of the Amount of Tracer Activity Added } \\
\sigma_{\mathrm{STS}}= & \text { Standard Deviation of the Amount of Tracer Activity Taken for } \\
& \text { Stock Tracer Solution (provided with certificates received with } \\
& \text { standards) } \\
\sigma_{\mathrm{MAss}}= & \text { Standard Deviation of the Mass of Standard Solution Used to } \\
\sigma_{\mathrm{T}} & \text { Prepare Stock Tracer Solution } \\
\sigma_{\mathrm{DL}}= & \text { Standard Deviation of the Volume(s) of the Dilution(s) Made to } \\
& \text { Prepare the Working Tracer Solution } \\
\sigma_{\mathrm{VOL}}= & \text { Standard Deviation of the Volume of the Stock Tracer Solution } \\
\sigma_{\mathrm{ALI}}= & \text { Standard Deviation(s) of the Aliquot(s) of Tracer Solution(s) } \\
& \text { Diluted to Prepare Working Tracer Solution } \\
\mathrm{C}_{\mathrm{GT}}= & \text { Gross Count of Tracer } \\
\mathrm{T}_{\mathrm{GT}}^{2}= & \text { Square of Tracer CountTime (minutes) } \\
\mathrm{C}_{\mathrm{TB}}= & \text { Background Count of Tracer [Area or Region of Interest (ROI)] } \\
\mathrm{T}_{\mathrm{TB}}^{2}= & \text { Square of Background CountTime (minutes) } \\
\mathrm{R}= & \text { Tracer Recovery } \\
\mathrm{NCR}_{\mathrm{T}}= & \text { Net Count Rate of the Tracer } \\
\mathrm{EFF}^{2}= & \text { Detector Efficiency } \\
\mathrm{AMT}_{\mathrm{T}}= & \text { Amount of Tracer Activity Added (dpm) } \\
\mathrm{STS}_{\mathrm{T}}= & \text { Amount of Tracer Activity (dpm) in Stock Tracer Solution } \\
\mathrm{MASS}_{\mathrm{T}}= & \text { Mass (grams) of Standard Solution Used to Prepare Stock Tracer } \\
& \text { Solution } \\
\mathrm{VOL}_{\mathrm{T}}= & \text { Volume of Tracer Solution Added } \\
\mathrm{DIL}_{\mathrm{T}}= & \text { Volume(s) of Dilution(s) Made to Prepare the Working Tracer } \\
\mathrm{ALI}_{\mathrm{T}}= & \text { Solution } \\
& \text { Sliquot(s) of Tracer Solution(s) Taken to Prepare Serial Tracer } \\
& \text { Solution Dilution(s) }
\end{aligned}
$$

Note: Certificates, such as those from NIST, may give two or even three sigma uncertainty. Only one sigma should be used for $\sigma_{\text {STS }}$. $^{\circ}$ 
Calculation of Recovery and $\mathrm{RE}_{\mathrm{R}}$ - Gravimetric - Method Blank Corrected Sample Concentrations)

$$
\begin{aligned}
& R=\frac{W T_{c}}{\operatorname{CONC}_{c S} * V O L_{c s}} \\
& \left(R E_{R}\right)^{2}=\left(\frac{\sigma_{R}}{R}\right)^{2}=\left(\frac{\sigma_{P P T . W T .}}{\text { PPT. WT. }}\right)^{2}+\left(\frac{\sigma_{C O N c_{c S}}}{\operatorname{CONC} c S}\right)^{2}+\left(\frac{\sigma_{V O L_{C S}}}{V O L_{C S}}\right)^{2} \\
& R E_{R}=\sqrt{\left(\frac{\sigma_{R}}{R}\right)^{2}} \\
& \text { Where: } \quad \mathrm{R}=\text { Carrier Recovery } \\
& \mathrm{RE}_{\mathrm{R}}=\text { Relative Error in Recovery } \\
& W T_{c}=\text { Weight of Carrier Present in Final Precipitate } \\
& \mathrm{CONC}_{\mathrm{cs}}=\text { Concentration of Carrier Solution } \\
& \mathrm{VOL}_{\mathrm{cs}}=\text { Volume of Carrier Solution Added } \\
& \sigma_{\text {PPT. wr. }}=\text { Standard Deviation in Weight of Precipitate } \\
& \sigma_{\text {CoNC }}=\text { Standard Deviation in Carrier Concentration } \\
& \sigma_{\mathrm{voL}}=\text { Standard Deviation in Carrier Volume } \\
& \text { PPT. WT. }=\text { Weight of Final Carrier Precipitate }
\end{aligned}
$$

\section{Counting Uncertainty (CU) at the 95\% Confidence Level}

$$
C U_{2 \sigma}=\frac{1.96 *\left(\sigma_{N_{C R}}\right)}{2.22 * E F F * A L I * R * A B N_{S} * e^{-\lambda t} * C F}
$$

Where:

$$
\begin{aligned}
\sigma_{\mathrm{NCR}_{s}}= & \text { Standard Deviation of the Net Sample Count Rate } \\
\mathrm{EFF}= & \text { Detector Efficiency } \\
\mathrm{ALI}= & \text { Sample Aliquot Volume or Mass } \\
\mathrm{R}= & \text { Sample Tracer/Carrier Recovery } \\
\mathrm{ABN}_{\mathrm{S}}= & \text { Abundance Fraction of the Emissions Used for Analyte } \\
& \text { Identification/Quantification } \\
\lambda= & \text { Analyte Decay Constant }-\ln 2 /(\text { half-life) [Same units as the } \\
& \text { half-life used to compute } \lambda] \\
\mathrm{t}= & \text { Time from Sample Collection To Radionuclide Separation or } \\
& \text { Mid-point of CountTime (Same units as half-life) } \\
\mathrm{CF}= & \text { Other Correction Factors as Appropriate (i.e., Ingrowth } \\
& \text { factor, Self-absorption Factor, etc.) }
\end{aligned}
$$


Sample Activity Concentration Total Propagated Uncertainty (TPU)-(Method Blank Corrected Sample Concentrations)

$T P U_{1 \sigma}=\sigma_{A C T}=\frac{\left.\sqrt{\sigma_{N C R_{S}}^{2}+(N C R)^{2} *\left(R E_{E F F}^{2}+R E_{A L I}^{2}+R E_{R}^{2}+\Sigma R E_{C F}^{2}\right.}\right)}{2.22 * E F F * A L I * R * A B N_{S} * e^{-\lambda t} * C F}$

Where: $\quad$ EFF $=$ Detector Efficiency

$\mathrm{ALI}=$ Sample Aliquot Volume or Mass

$\mathrm{R}=$ Sample Tracer/Carrier Recovery

$A B N_{S}=$ Abundance Fraction of the Emissions Used for Identification/Quantification

$\sigma_{\mathrm{NCR}}^{2}=$ Variance of the Net Sample Count Rate

NCR $=$ Net Sample Count Rate

$\mathrm{RE}_{\mathrm{EFF}}^{2}=$ Square of the Relative Error of the Efficiency Term

$\mathrm{RE}_{\mathrm{ALI}}^{2}=$ Square of the Relative Error of the Aliquot

$\mathrm{RE}_{\mathrm{R}}^{2}=$ Square of the Relative Error of the Sample Recovery

$\mathrm{RE}_{\mathrm{CF}}^{2}=$ Square of the Relative Error of Other Correction Factors

$\lambda=$ Analyte Decay Constant - In 2/(half-life) [Same units as the half-life used to compute $\lambda]$

$t=$ Time from Sample Collection to Radionuclide Separation or Mid-Point of Count Time (Same units as half-life)

$\mathrm{CF}=$ Other Correction Factors as Appropriate (i.e., Ingrowth factor, Self-absorption Factor, etc.) 
Sample Activity Concentration - Sample Concentrations Without Blank Subtraction

$$
A C T_{B}=\frac{N B C R_{S}}{2.22 * E F F * A L I * R * A B N_{S} * e^{-\lambda t} * C F}
$$

Where: $\quad \mathrm{ACT}_{\mathrm{B}}=$ Sample Activity Concentration Without Method Blank Subtraction

$\mathrm{NBCR}_{\mathrm{S}}=$ Net Sample Background-Corrected Count Rate

$2.22=$ Factor for Converting dpm to $\mathrm{pCi}$

$\mathrm{EFF}=$ Detector Efficiency

ALI $=$ Sample Aliquot Volume or Mass

$\mathrm{ABN}_{\mathrm{S}}=$ Abundance Fraction of the Emissions Used for Identification/Quantification

$\mathrm{R}=$ Sample Tracer/Carrier Recovery

$\lambda=$ Analyte Decay Constant $-\ln 2 /$ (half-life) [Same units as the half-life used to compute $\lambda$ ]

$\mathrm{t}=$ Time from Sample Collection to Radionuclide Separation or Mid-point of Count Time (Same units as half-life)

$\mathrm{CF}=$ Other Correction Factors as Appropriate (i.e., Ingrowth factor, Self-absorption Factor, etc.) 

Net Sample Count Rate $\left(\mathrm{NBCR}_{\mathrm{s}}\right)$ and $\mathrm{q}_{\mathrm{BCR}}$ - Sample Concentrations Without Blank
Subtraction

$$
N B C R_{S}=\left(\frac{C_{G S}}{T_{G S}}-\frac{C_{S B}}{T_{S B}}\right)
$$

Where:

$$
\begin{aligned}
\mathrm{NBCR}_{\mathrm{S}} & =\text { Net Background Corrected Count Rate } \\
\mathrm{C}_{\mathrm{GS}} & =\text { Sample Counts } \\
\mathrm{T}_{\mathrm{GS}} & =\text { Sample Count Time (minutes) } \\
\mathrm{C}_{\mathrm{SB}} & =\text { Background Counts } \\
\mathrm{T}_{\mathrm{SB}} & =\text { Background Count Time (minutes) }
\end{aligned}
$$

\section{$\sigma_{\mathrm{NBCR}_{\mathrm{s}}}$}

$$
\sigma_{N B C R_{S}}=\left[\frac{C_{G S}}{T_{G S}^{2}}+\frac{C_{S B}}{T_{S B}^{2}}\right]^{\frac{1}{2}}
$$


Calculation of Minimum Detectable Concentration (MDC) - general formula

$$
M D C=\frac{4.65 * \sqrt{b / T}}{K}+\frac{2.71}{K * T}
$$

Where:

$$
\begin{aligned}
& \mathrm{b}=\text { background count rate } \\
& \mathrm{T}=\text { Sample Count Time (minutes) } \\
& \mathrm{K}=\text { instrument-specific and sample-specific correction factors } \\
& \text { (e.g. } \left.\mathrm{ALI} * \mathrm{e}^{-\lambda \mathrm{t}} * \mathrm{R} * \mathrm{EFF}_{\mathrm{S}} * \mathrm{ABN}_{\mathrm{S}}\right)
\end{aligned}
$$

In using the above equation, the background and sample count times are either equivalent, or the background count time is greater than sample count time

Calculation of Recovery and $R E_{R}$ - Radiometric - Sample Concentrations Without Blank Subtraction

$$
R=\frac{\left(\frac{C_{G T}}{T_{G T}}-\frac{C_{T B}}{T_{T B}}\right)}{E E F * A B N_{T} * A M T_{T}}=\frac{N C R_{T}}{E F F * A B N_{T} * A M T_{T}}
$$

Where:

$$
\begin{aligned}
\mathrm{R} & =\text { Tracer Recovery } \\
\mathrm{C}_{\mathrm{GT}} & =\text { Gross Count of Tracer } \\
\mathrm{T}_{\mathrm{GT}} & =\text { Tracer CountTime (minutes) } \\
\mathrm{C}_{\mathrm{TB}} & =\text { Background Count of Tracer Region of Interest (ROI) } \\
\mathrm{T}_{\mathrm{TB}} & =\text { Background CountTime (minutes) } \\
\mathrm{EFF}= & \text { Detector Efficiency } \\
\mathrm{AMT}_{\mathrm{T}}= & \text { Amount of Tracer Activity Added (dpm) } \\
\mathrm{NCR}_{\mathrm{T}}= & \text { Net Count Rate of Tracer (cpm) } \\
\mathrm{ABN}_{\mathrm{T}}= & \text { Abundance Fraction of the Tracer Emissions used for } \\
& \text { Quantification of the Tracer }
\end{aligned}
$$


$\mathbf{R E}_{\mathrm{R}}$ - Radiometric - Sample Concentrations Without Blank Subtraction

$$
\begin{aligned}
& \left(R E_{R}\right)^{2}=\left(\frac{\sigma_{R}}{R}\right)^{2}=\left(\frac{\sigma_{N C R_{T}}}{N C R_{T}}\right)^{2}+\left(\frac{\sigma_{E F F}}{E F F}\right)^{2}+\left(\frac{\sigma_{A M T_{T}}}{A M T_{T}}\right)^{2} \\
& \left(\frac{\sigma_{N C R_{T}}}{N C R_{T}}\right)^{2}=\frac{\left(\frac{C_{G T}}{T_{G T}^{2}}+\frac{C_{T B}}{T_{T B}^{2}}\right)}{N C R_{T}^{2}} \\
& \left(\frac{\sigma_{A M T_{T}}}{A M T_{T}}\right)^{2}=\left(\frac{\sigma_{S T S_{T}}}{S T S_{T}}\right)^{2}+\left(\frac{\sigma_{M A S S_{T}}}{M A S S_{T}}\right)^{2}+\left(\frac{\sigma_{V O L_{T}}}{V O L_{T}}\right)^{2}+\Sigma\left(\frac{\sigma_{D I L_{T}}}{D I L_{T}}\right)^{2}+\Sigma\left(\frac{\sigma_{A L I_{T}}}{A L I_{T}}\right)^{2} \\
& R E_{R}=\sqrt{\left(\frac{\sigma_{R}}{R}\right)^{2}} \\
& \text { Where: } \quad R_{R}=\text { Relative Error of the Tracer Recovery } \\
& \sigma_{R}=\text { Standard Deviation of the Tracer Recovery } \\
& \sigma_{\mathrm{NCR}}=\text { Standard Deviation of the Tracer's Net Count Rate } \\
& \sigma_{\mathrm{EFF}}=\text { Standard Deviation of the Detector Efficiency } \\
& \sigma_{\mathrm{AMT}_{\mathrm{T}}}=\text { Standard Deviation of the Amount of Tracer Activity Added } \\
& \sigma_{\mathrm{STS}}^{\mathrm{T}}=\text { Standard Deviation of the Amount of Tracer Activity Taken for } \\
& \text { Stock Tracer Solution (provided with certificates received with } \\
& \text { standards) } \\
& \sigma_{\text {MASs }}=\text { Standard Deviation of the Mass of Standard Solution Used to } \\
& \text { Prepare Stock Tracer Solution } \\
& \sigma_{\mathrm{DI}_{\mathrm{T}}}=\text { Standard Deviation of the Volume(s) of the Dilution(s) Made to } \\
& \text { Prepare the Working Tracer Solution } \\
& \sigma_{\mathrm{VOL}_{\mathrm{T}}}=\begin{array}{l}
\text { Standard Deviation of the Volume of the Stock Tracer } \\
\text { Solution }
\end{array} \\
& \sigma_{\text {ALI }_{\mathrm{T}}}=\text { Standard Deviation(s) of the Aliquot(s) of Tracer Solution(s) } \\
& \text { Diluted to Prepare Tracer Working Solution } \\
& \mathrm{C}_{\mathrm{GT}}=\text { Gross Count of Tracer } \\
& \mathrm{T}_{\mathrm{GT}}^{2}=\text { Square of Tracer Count Time } \\
& \mathrm{C}_{\mathrm{TB}}=\text { Background Count of Tracer ROI } \\
& \mathrm{T}_{\mathrm{TB}}^{2}=\text { Square of Background Count Time } \\
& \mathrm{R}=\text { Tracer Recovery } \\
& \mathrm{NCR}_{\mathrm{T}}=\text { Net Count Rate of the Tracer } \\
& \mathrm{EFF}=\text { Detector Efficiency } \\
& \mathrm{AMT}_{\mathrm{T}}=\text { Amount of Tracer Activity Added (dpm) } \\
& \mathrm{STS}_{\mathrm{T}}=\text { Amount of Tracer Activity (dpm) in Stock Tracer Solution } \\
& \text { MASS }_{\mathrm{T}}=\text { Mass (grams) of Standard Solution Used to Prepare Stock Tracer } \\
& \text { Solution } \\
& \mathrm{VOL}_{\mathrm{T}}=\text { Volume of Tracer Solution Added } \\
& \mathrm{DIL}_{\mathrm{T}}=\text { Volume(s) of Dilution(s) Made to Prepare the Working Tracer } \\
& \text { Solution } \\
& \mathrm{ALI}_{\mathrm{T}}=\text { Aliquot(s) of Tracer Solution(s) Taken to Prepare Serial Tracer } \\
& \text { Solution Dilution(s) }
\end{aligned}
$$

Note: Certificates, such as those from NIST, may give two or even three sigma uncertainty. Only one sigma should be used for $\sigma_{\text {STs }}$. 
Calculation of Recovery and $\mathrm{RE}_{\mathrm{R}}$ - Gravimetric - Sample Concentrations Without Blank Subtraction

$$
\begin{gathered}
R=\frac{W T_{c}}{\operatorname{CONC}_{C S} * V O I_{C S}} \\
\left(R E_{R}\right)^{2}=\left(\frac{\sigma_{R}}{R}\right)^{2}=\left(\frac{\sigma_{P P T . W T .}}{P P T . W T .}\right)^{2}+\left(\frac{\sigma_{C O N C_{C S}}}{\left.\operatorname{CONC_{CS}}\right)^{2}+\left(\frac{\sigma_{V O I_{C S}}}{V O I_{C S}}\right)^{2}}\right. \\
R E_{R}=\sqrt{\left(\frac{\sigma_{R}}{R}\right)^{2}}
\end{gathered}
$$

Where: $\quad R=$ Carrier Recovery

$R E_{R}=$ Relative Error in Recovery

$\mathrm{WT}_{\mathrm{C}}=$ Weight of Carrier Present in Final Precipitate

$\mathrm{CONC}_{\mathrm{CS}}=$ Concentration of Carrier Solution

$\mathrm{VOL}_{\mathrm{Cs}}=$ Volume of Carrier Solution Added

$\sigma_{\text {pPT. wr. }}=$ Standard Deviation in Weight of Precipitate

$\sigma_{\text {CoNC }}=$ Standard Deviation in Carrier Concentration

$\sigma_{\mathrm{voL}}=$ Standard Deviation in Carrier Volume

PPT. WT. $=$ Weight of Final Carrier Precipitate

Counting Uncertainty (CU) at the $95 \%$ Confidence Level

$$
C U_{B_{2 \sigma}}=\frac{1.96 *\left(\sigma_{N B C R_{S}}\right)}{2.22 * E F F * A I I * R * A B N_{S} * e^{-\lambda t} * C F}
$$

Where:

$\sigma_{\mathrm{NBCR}_{\mathrm{S}}}=$ Standard Deviation of the Net Background Corrected Count

$\mathrm{EFF}=$ Detector Efficiency

ALI = Sample Aliquot Volume or Mass

$\mathrm{R}=$ Sample Tracer/Carrier Recovery

$\mathrm{ABN}_{\mathrm{S}}=$ Abundance Fraction of the Emissions Used for Identification/Quantification

$\lambda=$ Analyte Decay Constant $-\ln 2 /($ half-life) [Same units as the half-life used to compute $\lambda]$

$\mathrm{t}=$ 'Time from Sample Collection To Radionuclide Separation or Mid-point of Count Time (Same units as half-life)

$\mathrm{CF}=$ Other Correction Factors as Appropriate (i.e., Ingrowth factor, Self-absorption Factor, etc.) 
Sample Activity Concentration Total Propagated Uncertainty (TPU)Sample Concentrations Without Blank Subtraction

$$
T P U_{B_{1 \sigma}}=\sigma_{A C T}=\frac{\left.\sqrt{\sigma_{N B C R_{S}}^{2}+\left(N B C R_{S}\right)^{2} *\left(R E_{E F F}^{2}+R E_{A L I}^{2}+R E_{R}^{2}+\Sigma R E_{C F}^{2}\right.}\right)}{2.22 * E F F * A L I * R * A B N_{S} * e^{-\lambda t} * C F}
$$

Where:

$$
\begin{aligned}
\mathrm{EFF}= & \text { Detector Efficiency } \\
\mathrm{ALI}= & \text { Sample Aliquot Volume or Mass } \\
\mathrm{R}= & \text { Sample Tracer/Carrier Recovery } \\
\mathrm{ABN}_{\mathrm{S}}= & \text { Abundance Fraction of the Emissions Used for Analyte } \\
& \text { Identification/Quantification } \\
\sigma_{\mathrm{NBCR}}^{2}= & \text { Variance of the Net Background Corrected Count Rate } \\
\mathrm{NBCR}_{\mathrm{S}}= & \text { Net Background Corrected Count Rate } \\
\mathrm{RE}_{\mathrm{EFF}}^{2}= & \text { Square of the Relative Error of the Efficiency Term } \\
\mathrm{RE}_{\mathrm{ALI}}^{2}= & \text { Square of the Relative Error of the Aliquot } \\
\mathrm{RE}_{\mathrm{R}}^{2}= & \text { Square of the Relative Error of the Sample Recovery } \\
\mathrm{RE}_{\mathrm{CF}}^{2}= & \text { Square of the Relative Error of Other Correction Factors } \\
\lambda= & \text { Analyte Decay Constant }- \text { ln } 2 / \text { (half-life) [Same units as the } \\
& \text { half-life used to compute } \lambda] \\
\mathrm{t}= & \text { Time from Sample Collection to Radionuclide Separation or } \\
& \text { Mid-Point of Count Time (Same units as half-life) } \\
\mathrm{CF}= & \text { Other Correction Factors as Appropriate (i.e., Ingrowth } \\
& \text { factor, Self-absorption Factor, etc.) }
\end{aligned}
$$

NOTE:

For methods using a tracer or carrier, the inclusion of efficiency and recovery terms in the equation above may result in overestimation of the TPU. 


\section{APPENDIX D \\ LISTING OF COMMON NUCLIDES/METHODOLOGIES}



D-3

Source of radionuclide identities: DOE National Sample Tracking System, FY 1994

\begin{tabular}{|c|c|c|c|c|c|c|c|c|c|c|c|c|}
\hline Analysis Type & \multicolumn{6}{|c|}{ Matrix } & \multicolumn{5}{|c|}{ Instrumentation } & \multirow[t]{2}{*}{ Source } \\
\hline : & 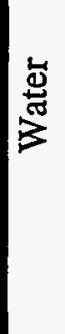 & $\bar{\varnothing}$ & $\begin{array}{l}\frac{\pi}{4} \\
\frac{2}{1}\end{array}$ & ஜํㅠㅇ & 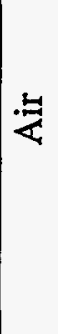 & 密 & 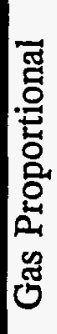 & 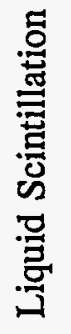 & 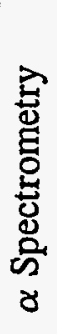 & 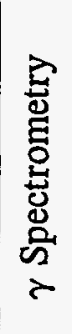 & 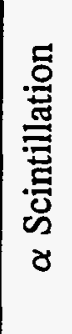 & \\
\hline Gross $\alpha / \beta$ & $\mathrm{X}$ & $\mathrm{x}$ & & & & $\cdot$ & $\mathrm{x}$ & & & & & $2 \& 4$ \\
\hline${ }^{3} \mathrm{H}$ & $\mathrm{x}$ & $\mathrm{x}$ & & & & $\mathrm{X}$ & 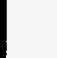 & $\mathrm{x}$ & & & & 2 \\
\hline Pu isotopic & $\mathrm{x}$ & $\mathrm{X}$ & $\mathrm{X}$ & & $\mathrm{x}$ & $\mathrm{X}$ & & & $\mathrm{x}$ & & . & 2 \\
\hline U Isotopic & $\mathrm{x}$ & $\mathrm{X}$ & $\mathrm{x}$ & & $\mathrm{X}$ & $\mathrm{X}$ & & & $x$ & & & 2 \\
\hline${ }^{241} \mathrm{Am}$ & $\mathrm{x}$ & $\mathrm{x}$ & $\mathrm{x}$ & & & $\mathrm{X}$ & 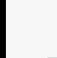 & & $\mathrm{x}$ & & & 2 \\
\hline${ }^{90} \mathrm{Sr}$ & $\mathrm{x}$ & & $\mathrm{x}$ & $\mathrm{x}$ & & & $\mathrm{x}$ & $\mathrm{x}$ & & & & $2 \& 4$ \\
\hline${ }^{223,224,226} \mathrm{Ra}$ & $\mathrm{x}$ & & & & & & 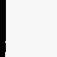 & & & & $\mathrm{X}$ & 3 \\
\hline${ }^{226} \mathrm{Ra}$ & $\mathrm{x}$ & & & $\mathrm{X}$ & & & & & & & $\mathrm{X}$ & 2 \\
\hline${ }^{228} \mathrm{Ra}$ & $\mathrm{x}$ & & & & & & $\mathrm{X}$ & & & & & 2 \\
\hline${ }^{97} \mathrm{Tc}$ & $\mathrm{x}$ & & & & & & & $\mathrm{x}$ & & & & 1 \\
\hline Th Isotopic & $\mathrm{x}$ & $\mathrm{x}$ & $\mathrm{x}$ & & $\mathrm{x}$ & $\mathrm{X}$ & $\mathrm{x}$ & & $\mathrm{x}$ & & & 2 \\
\hline${ }^{129} \mathrm{I}$ & $\mathrm{X}$ & & & & & & 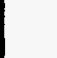 & & & $\mathrm{x}$ & & 4 \\
\hline Cm Isotopic & $\mathrm{x}$ & & & & & & $\mathrm{X}$ & & & & & 3 \\
\hline${ }^{89} \mathrm{Sr}$ & $\mathrm{x}$ & & $\mathrm{X}$ & $\mathrm{x}$ & & & $\mathrm{x}$ & $\mathrm{X}$ & & & & $2 \& 4$ \\
\hline${ }^{14} \mathrm{C}$ & $\mathrm{x}$ & & & . & & & $\mathrm{x}$ & & & & & 2 \\
\hline${ }^{237} \mathrm{~Np}$ & $\mathrm{x}$ & & & & & & $\mathrm{x}$ & & & & $\mathrm{X}$ & 3 \\
\hline${ }^{222} \mathrm{Rn}$ & & & & & & & & & & & & \\
\hline${ }^{210} \mathrm{~Pb}$ & $\mathrm{x}$ & & & $\mathrm{x}$ & & $\mathrm{x}$ & $\mathrm{x}$ & & & & & 2 \\
\hline${ }^{210} \mathrm{Po}$ & & & & & & $\mathrm{x}$ & & & $\mathrm{x}$ & & & 2 \\
\hline${ }^{79} \mathrm{Se}$ & $\mathrm{x}$ & & & & & & & $\mathrm{x}$ & & & & 4 \\
\hline $\begin{array}{l}\text { Gamma } \\
\text { Emitters }\end{array}$ & $\mathrm{x}$ & & & & & & & & & $\mathrm{x}$ & & 3 \\
\hline
\end{tabular}

Sources:

1. $\quad$ "Technetium-99 Analysis Using Extraction Chromatography"

2. "Eastern Environmental Radiation Facility Radiochemistry Procedures Manual"

3. - United States Environmental Protection Agency. Prescribed Procedures for Measurement of Radioactivity in Drinking Water. EPA-600/4-80-032.

4. DOE Methods for Evaluating Environmental and Waste Management Samples DOE/EM-O089T, Rev. 2, October, 1994. 


\section{APPENDIX E}

\section{RECOMMENDED SURVEILLANCE CONSIDERATIONS}

FOR GAMMA ALGORITHMS 

Radionuclide identification algorithms used by the laboratory should be evaluated during laboratory audits. Consideration should be given to the following:

i. the width of the energy-search windows for radionuclide identification to ensure a minimum of peak misidentifications,

ii. the criteria by which the laboratory evaluates sample-specific peak resolution,

iii. documentation of the source of the isotope identification library used by the source [Most software packages come with a master library which users can pare down to one more useful in day to day analyses.],

iv Training records of those persons(s) using the peak identification respective software,

v. SOP documentation of the parameters used by the software in the analysis of gamma spectra,

vi. existence of SOP documentation for the internal review of the reported data,

vii. If the software package is capable of using pre-programmed macros to automate the spectral analysis, evaluate the existence of SOP documentation of these macros,

viii. If the software package uses various flags to denote peak quality or analysis results, the analyst using the software should be able to explain the meaning of the flags and their impact on data quality. 